\title{
1 Evolutionary history of sexual differentiation mechanism in insects
}

2 Yasuhiko Chikami ${ }^{1,2}$, Miki Okuno ${ }^{3}$, Atsushi Toyoda ${ }^{4,5}$, Takehiko Itoh $^{6}$, Teruyuki

$3 \mathrm{Niimi}^{1,2} *$

$4 \quad{ }^{1}$ Division of Evolutionary Developmental Biology, National Institute for Basic

5 Biology, 38 Nishigonaka, Myodaiji, Okazaki, Aichi, 444-8585, Japan

$6 \quad{ }^{2}$ Department of Basic Biology, School of Life Science, The Graduate University for

7 Advanced Studies, SOKENDAI, 38 Nishigonaka, Myodaiji, Okazaki, Aichi, 444-

8 8585, Japan

$9 \quad{ }^{3}$ Division of Microbiology, Department of Infectious Medicine, Kurume University

10 School of Medicine, 67 Asahi-machi, Kurume, Fukuoka, 830-0011, Japan

$11{ }^{4}$ Comparative Genomics Laboratory, National Institute of Genetics, 1111 Yata,

12 Mishima, Shizuoka 411-8540, Japan

$13{ }^{5}$ Advanced Genomics Center, National Institute of Genetics, 1111 Yata, Mishima,

14 Shizuoka 411-8540, Japan

$15{ }^{6}$ School of Life Science and Technology, Tokyo Institute of Technology, 2-12-1

16 Ookayama, Meguro, Tokyo, 152-8550, Japan

17 *Corresponding author: Teruyuki Niimi

18 E-mail: niimi@nibb.ac.jp

\section{This PDF file includes:}

Main Text

Figures 1 to 6 


\section{Abstract}

Gain of alternative splicing gives rise to functional diversity in proteins and underlies the complexity and diversity of biological aspects. However, it is still not fully understood how alternatively spliced genes develop the functional novelty. To this end, we infer the evolutionary history of the doublesex gene, the key transcriptional factor in the sexual differentiation of arthropods. doublesex is controlled by sexspecific splicing and promotes both male and female differentiation in some holometabolan insects. In contrast, doublesex promotes only male differentiation in some hemimetabolan insects. Here, we investigate ancestral states of doublesex using Thermobia domestica belonging to Zygentoma, the sister group of winged insects. We find that doublesex of $T$. domestica expresses sex-specific isoforms but is only necessary for male differentiation of sexual morphology. This result ensures the hypothesis that doublesex was initially only used to promote male differentiation during insect evolution. However, T. domestica doublesex has a short female-specific region and upregulates the expression of vitellogenin homologs in females, suggesting that doublesex may have already controlled some aspects of feminization in the common ancestor of winged insects. Reconstruction of the ancestral sequence and prediction of the protein structure show that the female-specific isoform of doublesex has a long C-terminal disordered region in holometabolan insects, but not in nonholometabolan species. We propose that doublesex acquired a female-specific isoform and then underwent a change in the protein motif structure, which became essential for female differentiation in sexual dimorphisms.

\section{Keywords: sexual differentiation, alternative splicing, doublesex, insect,}

\section{Zygentoma}




\section{$48 \quad$ Introduction}

Sexual reproduction is widely used for transmitting genetic information from one to the next generation in Metazoa. For reproductive success, animals evolved diverse sex differences, i.e., sexual dimorphism, in morphology (Darwin 1871; Geddes and Thomson 1889) which underlie eco-evolutionary dynamics such as extinction rate and interspecific interaction (Fryxell et al. 2019). In the last three decades, the genetic pathways that create sex and sexual dimorphism have been elucidated in many animal species. Surprisingly, despite having a single origin (Beukeboom and Perrin 2014), these pathways have undergone extensive changes during animal evolution (Wilkins 1995; Bachtrog et al. 2014; Bopp et al. 2014; Herpin and Schartl 2015).

The diversity has been attributed to differences in the composition of the regulatory cascades. For example, in eutherians such as mice and humans, the master regulator of sex is Sex-determining region $Y($ Sry), a member of the High Mobility Group (HMG)-box transcriptional factor family (Gubbay et al. 1990; Sinclair et al. 1990; Koopman et al. 1991; Miyawaki et al. 2020), while DM domain gene on the $Y$ chromosome (dmy) of the doublesex and mab-3 related transcriptional factor (DMRT) family is the master sex-determining regulator in the medaka fish (Matsuda et al. 2002; Nanda et al. 2002). Diversification of the pathway governing sex determination/differentiation are largely based on differences in their gene repertoires (e.g., Hasselmann et al. 2008; Hattori et al. 2010; Sato et al. 2010; Takehana et al. 2014). In contrast, it has recently been discovered that the mechanisms of sexual 
differentiation in Pterygota, i.e., winged insects, differ in outputs of the gene cascade, e.g., the promotion masculinization or feminization.

Sexually dimorphic morphology in Pterygota is formed during postembryonic development. doublesex $(d s x)$, a member of the DMRT family, acts as a global regulator at the bottom of the cascade to govern over sex differentiation (Kopp, 2012; Verhulst and van de Zande, 2015). In many pterygote insects studied, $d s x$ is controlled by sex-specific splicing. In Diptera, Coleoptera, and Lepidoptera, sex-specific Dsx protein variants are essential for promoting either male or female differentiation in sexual dimorphism (e.g., Hildreth 1965; Burtis and Baker 1989; Ohbayashi et al. 2001; Kijimoto et al. 2012; Ito et al. 2013; Shukla and Palli 2012; Gotoh et al. 2016; $\mathrm{Xu}$ et al. 2017). For example, in the fruit fly Drosophila melanogaster, $d s x$ is required to realize sex differences in external genitalia and foreleg bristle rows, while $d s x$ mutants show an intersexual phenotype in these traits because both male and female differentiation are inhibited (Hildreth and Lucchesi 1963; Hildreth 1965). However, in the sawfly Athalia rosae (Mine et al. 2017, 2021), the silverleaf whitefly Bemisia tabaci (Guo et al. 2018), the brown planthopper Nilaparvata lugens (Zhuo et al. 2018), the German cockroach Blattella germanica (Wexler et al. 2019), and the damselfly Ischnura senegalensis (Takahashi et al. 2019, 2021), $d s x$ has sex-specific isoforms and is responsible for male differentiation of morphological traits during postembryonic development, but not needed for female differentiation. Thus, despite expressing sex-specific isoforms, $d s x$ 's role in sexual differentiation in Pterygota is different, as it controls both male and female differentiation or only male differentiation for sexual morphogenesis. 

morphological traits in the water flea Daphnia magna. Wexler et al. (2019) proposed a stepwise evolution in which $d s x$ had acquired sex-specific isoforms and later had become essential for female differentiation. However, roles of $d s x$ are more diverse than expected. In Hymenoptera, $d s x$ is involved in female differentiation of reproductive organs in the honeybee Apis mellifera (Roth et al. 2019), while $d s x$ is non-essential for female differentiation in the sawfly At. rosae (Mine et al. 2017, 2021). In the milkweed bug Oncopeltus fasciatus, $d s x$ is involved in both female and male differentiation of the genital organs (Just et al. 2021). dsx in Be. tabaci positively regulates the expression of a yolk precursor gene vitellogenin in females while it is not essential for female morphology (Guo et al. 2018), implying that $d s x$ has different evolutionary history of $d s x$ in Pterygota is still a challenging task. Also, it is unclear what factors led to the feminizing roles of $d s x$ (Hopkins and Kopp 2021). information about outgroups more closely related to Pterygota may be the reason for the gap of understanding of how $d s x$ evolved from a monofunctional to a bifunctional

112 regulator in arthropods. In an attempt to close this gap, we decided to include the firebrat Thermobia domestica (Zygentoma) in our analysis of $d s x$. Zygentoma is the

114 sister group of Pterygota (Misof et al. 2014), does not copulate, and displays simple

115 sexual dimorphisms, i.e., non-aedeagus male penises and female ovipositors 
117 2018). Otherwise, there is little difference in morphology between females and males,

118 as Darwin (1871: 348) noted, "The sexes do not differ." These features suggest that

119 the level of sex differentiation in this species is very simple and likely to be ancestral.

120 Thus, Zygentoma presents an ideal model for investigating the ancestral state of $d s x$ in

121 Pterygota. In this study, we investigated $d s x$ in T. domestica and analyzed its

122 functions in sexual differentiation. Also, we carried out the phylogenetic analysis,

123 ancestral sequence reconstruction, and protein structure prediction to infer the

124 evolutionary history of $d s x$.

\section{Results and Discussion}

\section{Molecular evolution of $d s x$ homologs and gene duplication of $d s x$ in insects}

Five doublesex $(d s x)$ homologs were found in the transcriptome database of the firebrat Thermobia domestica. To identify which of them corresponds to the $d s x$ ortholog in $T$. domestica, we compared these to $d s x$ homologs found in transcriptome/genome/protein databases of various arthropods and vertebrates (supplementary table 1) and performed molecular phylogenetic analyses based on the amino acid sequences of their DNA-binding domains. As a result, the pancrustacean $d s x$ was grouped into a distinct clade from the other DMRT family genes (fig. 1A). Within this clade, four subclades were recognized: Insect Dsx Clade 1, Insect Dsx Clade 2, Entognatha Dsx Clade, and Crustacea Dsx Clade. Insect Dsx Clade 1 contained the $d s x$ found in Pterygota including Drosophila melanogaster. This clade also contained one of the $5 d s x$ homologs of $T$. domestica. We consider it likely that 
140 Clade also contained $d s x$ from a springtail (Collembola) and a dipluran insect

141 (Diplura). The Crustacea Dsx Clade contained $d s x$ from branchiopods, including

142 daphnids.

143 We found that several species of Zygentoma, Ephemeroptera (mayflies), and

144 Phasmatodea (stick insects) contain a $d s x$-like homolog of the Insect Dsx Clade 2 (fig.

$1451 \mathrm{~A}, \mathrm{~B})$ as well as the dsx ortholog of the Insect Dsx Clade 1. These findings indicate

146 that $d s x$ was duplicated before the divergence of Zygentoma and that the two paralogs

147 retained from the divergence of the pterygote insects until at least the divergence of

148 Eumetabola (= Hemiptera + Thysanoptera + Psocodea + Holometabola $).$ The

149 molecular evolution of $d s x$ has been inferred from $d s x$ of some pterygote insects,

150 mainly holometabolan insects (Wexler et al. 2014; Mawaribuchi et al. 2019), while

151 the presence of a $d s x$-like gene may have been overlooked in their analyses. Here, we

152 report that the genome of $T$. domestica also contains both $d s x$ and $d s x$-like, reflecting

153 the presumed ancestral state in Pterygota in terms of gene copy number of $d s x$. Gene

154 duplication generally leads to neo-/sub-functionalization to allow functional

155 diversification (c.f., Taylor and Raes 2004). In this study, we analyzed the expression

156 profiles and functions of $d s x$ as well as $d s x$-like in T. domestica. 


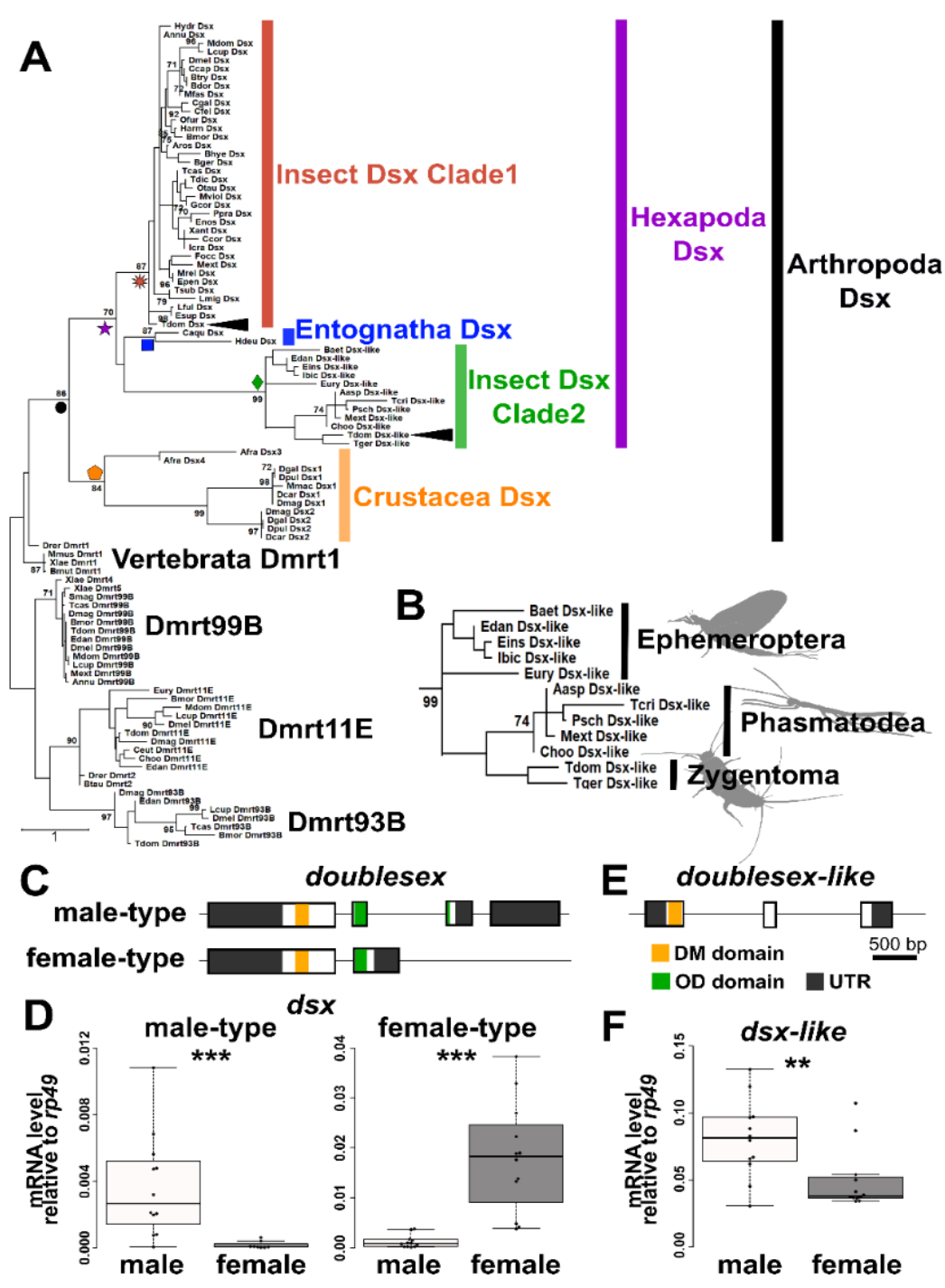

FIG. 1. Molecular phylogeny and structural features of Doublesex in Arthropoda and Vertebrata. (A) Molecular phylogeny of Doublesex and Mab-3 related transcriptional factors (DMRT). The phylogenetic analysis was based on amino acid sequences of the DNA binding domain (DM domain) of DMRT family and was performed by the MEGA $X$ after the multiple sequence alignment using the MAFFT software. The maximum-likelihood method was applied. 97 operational taxonomic units (OTUs) used for the phylogenetic analysis are listed in supplementary table 1. (B) Enlarged view of insect Dsx Clade2 ( $d s x$-like clade). The numerical value on each node is the bootstrap supporting value. Bootstrap values $<70$ are not shown. The node of each clade is indicated by colored shapes: black circle, Arthropoda Dsx; orange pentagon, Crustacea Dsx; purple star, Hexapoda Dsx; red sunburst, Insect Dsx Clade1; green diamond, Insect Dsx Clade2; blue square, Entognatha Dsx. (C) Exon-intron structures of $d s x$ in Thermobia domestica. The upper and lower schematic images show the gene structure of $d s x$ male-type and female-type, respectively. (D) Expression level of $d s x$ in males and females of $T$. domestica. (E) Exon-intron structures of dsx-like of $T$. domestica. (F) Expression level of $d s x$-like in males and females. The exon-intron structure is determined by mapping the mRNA sequence of each gene to the genome 
175

176

177

178

179

180

181

182

183

184

185

186

187

188

of $T$. domestica. The expression level (D and F) was measured by the RT-qPCR of $d s x$ and $d s x$-like in the adult fat body and is indicated as the relative values to the expression of the reference gene, ribosomal protein 49 (rp49). Each plot indicates the mRNA expression level of each individual. Total $N=20$ ( $d s x$ male-type), 23 ( $d s x$ female-type), and 24 (dsx-like). Results of Brunner-Munzel tests are indicated by asterisks: $* * P<0.01 ; * * * P<0.001$ and are described in supplementary table 2 .

\section{Sex-specific splicing of $d s x$ in Thermobia domestica}

Splicing of $d s x$ produces gives rise to sex-specific isoforms in all pterygote insects studied thus far, with the exception of the termite Reticulitermes speratus (Miyazaki et al. 2021), the silverleaf whitefly Bemisia tabaci (Guo et al. 2018), and the body louse Pediculus humanus (Wexler et al. 2019), suggesting that sex-specific splicing regulation of $d s x$ was acquired before the divergence of Pterygota. To examine this hypothesis, we investigated the expression profile of $d s x$ and $d s x$-like of T. domestica. Full-length mRNA sequences of $d s x$-like and $d s x$ in T. domestica were determined by the RNA-seq and rapid amplification of cDNA ends (RACE) methods. Then, we investigated the gene structures to map the mRNA sequences to our genome database. $d s x$ consists of five exons with two isoforms (fig. 1C): a long one (951 bp) and a short one (756 bp). RT-qPCR analysis showed that the long isoform and the short isoform were highly expressed in males and females, respectively (fig. 1D; Brunner-Munzel test, $P=1.75 \times 10^{-6}$ and $2.20 \times 10^{-16}$ in the long and the short isoforms). This fact indicates that $d s x$ is controlled by sex-specific splicing. We refer to the male-biased isoform as $d s x$ male-type and the female-biased isoform as $d s x$ female-type. The $d s x$-like is expressed about two-fold higher in males than in females (Brunner-Munzel test, $P=0.00924$ ) and has three exons but no sex-specific isoform (fig. 1E, F), showing that $d s x$-like is not regulated by sex-specific splicing. Our results give the further support that sex-specific splicing of $d s x$ already existed in the common ancestor of Pterygota and Zygentoma (= Dicondylia), which 
203

204

205

206

207

208

209

210

211

212

213

214

215

216

217

diverged 421 million years ago (Ma). Misof et al. (2014) estimated that the common ancestor of Daphnia and hexapods occurred at 508 Ma. Therefore, $d s x$ sex-specific splicing regulation is an ancient feature of insects that was acquired between 508 and $421 \mathrm{Ma}$ and has been conserved for $\sim 400$ million years in each taxon of Dicondylia.

\section{Function of $d s x$ for internal reproductive system and body size in T. domestica}

Deciphering the role of $d s x$ of $T$. domestica is essential for inferring the ancestral roles of $d s x$ in pterygote insects. Hence, we conducted a functional analysis of not only $d s x$ but also $d s x$-like since this paralog might also play a role in sexual differentiation.

To this end, we silenced $d s x$ and $d s x$-like by RNA interference (RNAi). We quantified the expression of $d s x$ and $d s x$-like in fat bodies of RNAi individuals by reverse transcriptional quantitative polymerase chain reaction (RT-qPCR). $d s x$ silencing in females and $d s x$-like silencing in both sexes showed significantly decreased expression of each target genes compared to their expression in enhanced green fluorescent protein (egfp) RNAi controls (Brunner-Munzel test, $P=0.0265$ in female $d s x, 4.40 \times 10^{-16}$ in male and female $d s x$-like; supplementary fig. 1A; supplementary table 2). The $d s x$ RNAi males did not show a significant effect on $d s x$ expression. Since it was suspected that outliers affected this result, we tested for outliers in $d s x$ RNAi males and found one outlier (supplementary table 3). The reanalysis removing the outlier showed that $d s x$ expression was significantly decreased in $d s x$ RNAi males (Brunner-Munzel test, $P=0.00545$; supplementary fig. 1B; supplementary table 2). Therefore, we concluded that $d s x$ and $d s x$-like dsRNAs can knock down each target gene. Also, $d s x$ RNAi had no effect on $d s x$-like expression and vice versa. 

silencing on sexual dimorphism such as body size (fig. 2A) and reproductive systems. dsx-like RNAi, and double knockdown did not show any histological differences in

like), the male seminal vesicle, which is a sperm storage organ and normally has a

supplementary table 5; generalized linear model, $P=0.00487)$. Silencing of dsx or

(fig. 2G; supplementary table 4).

249 focuses on the function of $d s x$ during postembryonic development. The lack of effect

250 of $d s x$ on the body size of $T$. domestica is consistent with studies in D. melanogaster 


\section{A}

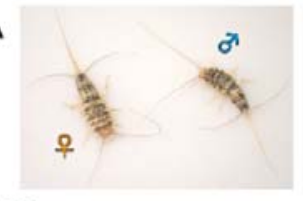

C
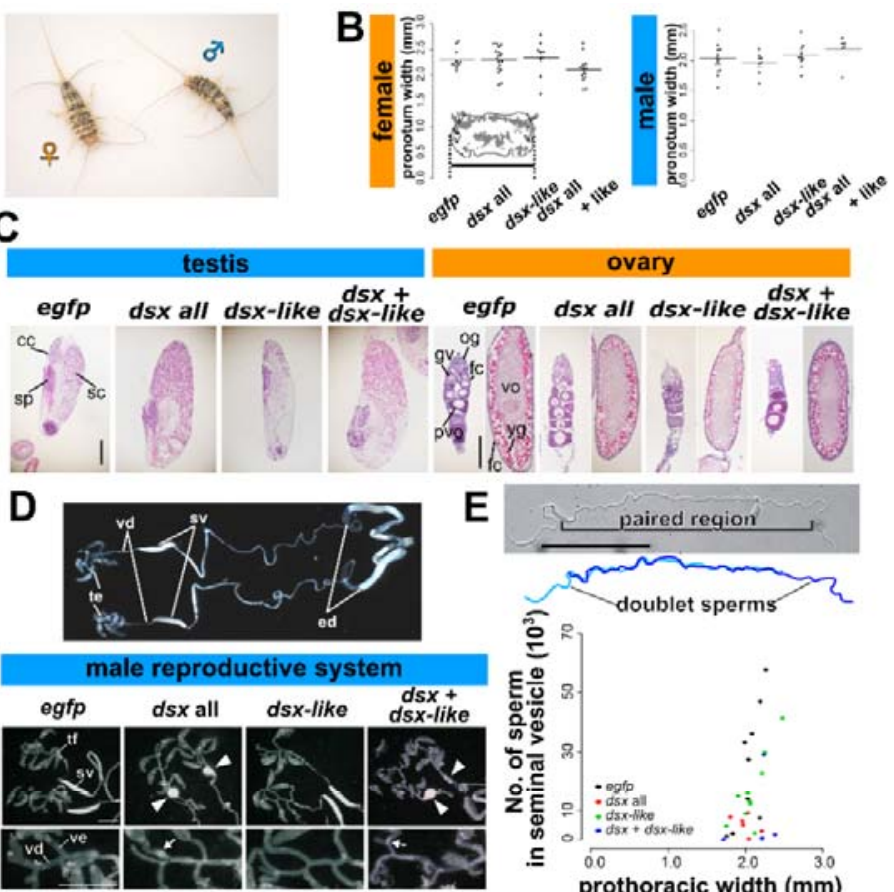

$\mathbf{F}$
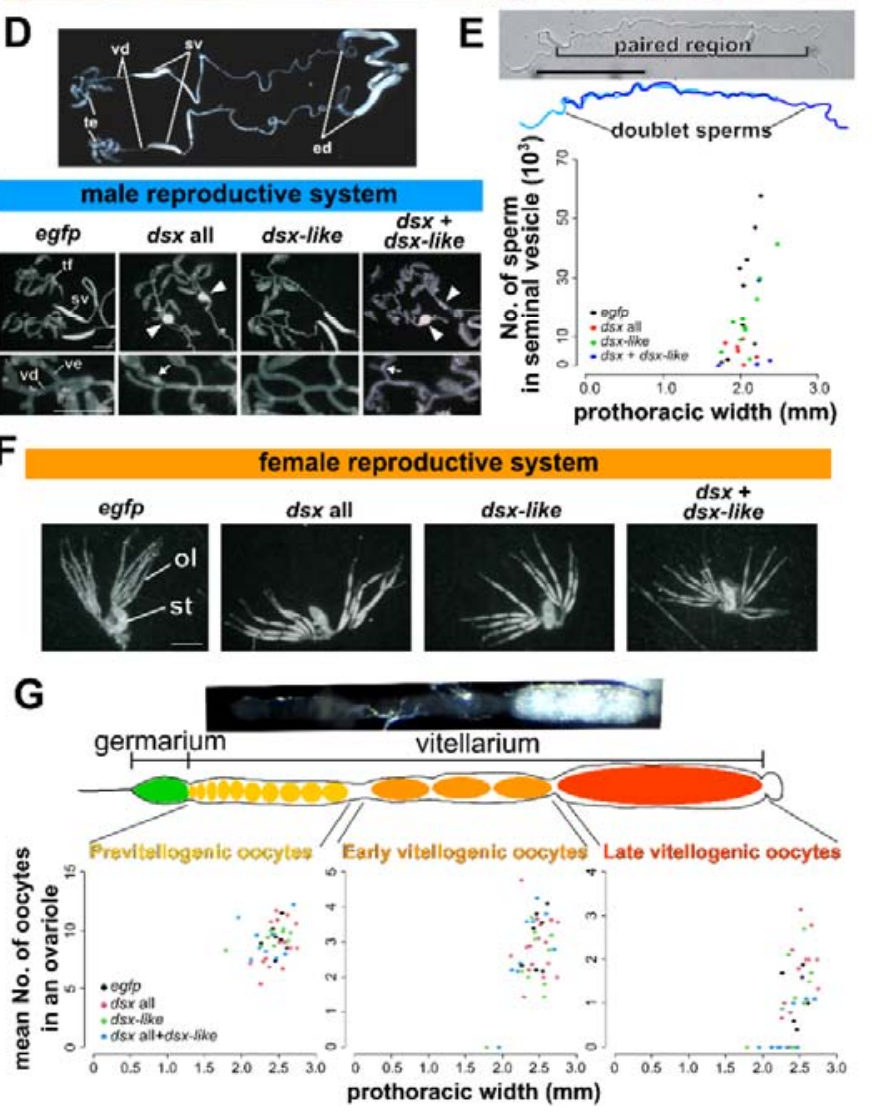

FIG. 2. Function of doublesex and doublesex-like for body size, internal reproductive system, and gametogenesis of Thermobia domestica. (A) A pair of T. domestica. The female looks much the same as the male. $(B)$ Body size of RNAi treatment groups. The pronotum (prothoracic tergum) width was used for the index of the body size. The graph shows mean \pm SE (standard error). The results of the generalized linear 
model (GLM) analysis show in supplementary table 4 (female) and 5(male). Any significant effect can be detected in the RNAi treatments. Total $N=49$ in females and 36 in males. $(C)$ Histology of gonads in the RNAi groups. Paraffin. HematoxylinEosin staining. In images of the ovary, the left and right panel in each treatment show germarium/previtellogenesis and vitellogenesis, respectively. (D) Effects of RNAi on male internal reproductive system. The upper photo shows the gross morphology of the reproductive systems in the non-treated male. The lower photos demonstrate the morphology of the RNAi males. The arrowheads show the rounded seminal vesicle. The lowest photos focused on the vas efferens. The arrows show the clogged sperm in the vas efferens. $(E)$ Sperm of RNAi males. The upper photo and figure are sperm morphology in the non-treated male. The sperm forms doublet in the seminal vesicle. The lower figure shows the sperm number of the RNAi males. The results of the GLM analysis show in supplementary table 5. The significant effect was detected in the $d s x$ RNAi treatment $(P=0.00487)$. Total $N=29$. $(F)$ Effects of RNAi on female internal reproductive system. $(G)$ Effects of the RNAi on oocyte number. The upper photo shows the ovariole of the non-treated female. The lower figures exhibit the number of oocytes in the RNAi females along with the oogenetic stages. The results of the GLM analysis show in supplementary table 4 . The number of the late vitellogenic oocytes was correlated with the pronotum width, although any significant effect can be detected in the RNAi treatments. Total $N=42$ in each stage. In each panel, the egfp, $d s x$ all, $d s x$-like and $d s x+d s x$-like indicates the egfp dsRNA injected group (control), $d s x$ sex-common region dsRNA injected group, $d s x$-like dsRNA injected group, and both $d s x$ sex-common region and $d s x$-like dsRNAs injected group, respectively. Each plot in $(B),(E)$, and $(G)$ indicates the value of each individual. cc, cystocyte; fc, follicle cell; gv, germinal vesicle; og, oogonia; ol, ovariole; pvo, previtellogenic oocyte; sc, spermatocyte; sp, sperm; st, spermatheca; sv, seminal vesicle; tf, testicular follicle; yg, yolk granule; ve, vas efferens; vd, vas deferens, vo, vitellogenic oocyte. Scales: $50 \mu \mathrm{m}(C) ; 10 \mu \mathrm{m}(E) ; 1000 \mu \mathrm{m}(D$ and $F)$.

\section{Function of $d s x$ for morphology in genital organs of T. domestica and evolution of}

\section{the function of $d s x$ for sexual morphogenesis in insects}

The sexually dimorphic morphology can be seen in the external genital organs, i.e., male penis and female ovipositor (fig. 3A). Males of T. domestica have unpaired small external genitalia on the abdominal segment IX. Females have an ovipositor consisted of two paired appendage-like structure on the abdominal segment VIII and IX. Males of the $d s x$ knockdown groups ( $d s x$ only and both $d s x$ and $d s x$-like RNAi) was transformed into two pairs of appendage-like structures resembling the female ovipositor (fig. 3B, C; Supplementary Material online). This effect was not observed 
299

300

301

302

303

304

305

306

in $d s x$-like RNAi males. Our results indicate that $d s x$ is essential for male differentiation of morphological traits in T. domestica. In contrast to males, our analysis showed that none of the RNAi treatments affected female ovipositors at external morphological, tissue, or cellular levels (fig. 3D, E; supplementary fig. 3; Supplementary Material online). We then measured the length of the female ovipositor in the RNAi-treated groups to examine the involvement of $d s x$ and $d s x$-like in the growth of female morphology. The results showed that $d s x$ and $d s x$-like RNAi had no significant effect on ovipositor length (fig. 3F, G; supplementary table 4).

The lack of effect of $d s x$ RNAi in females is due to that $d s x$ is not essential for female differentiation of morphology during postembryonic development or that $d s x$ knockdown is inefficient in the females. Compared to the knockdown efficiency of $d s x$ in the males ( $\sim 30 \%$ at median), that in the females is $\sim 50 \%$ (supplementary table 2). However, given that half of the female individuals in the RT-qPCR analysis in the fat body had lower $d s x$ expression than the minimum value of the control ones (supplementary fig. 1A), it can be assumed that $d s x$ expression is suppressed in a certain number of females used in each analysis. In addition, $d s x$ RNAi showed no effect on morphology in all 58 females (80 females including $d s x$ and $d s x$-like double RNAi) analyzed in this study. Thus, it is reasonable to conclude that $d s x$ is not essential for female differentiation of morphology during postembryonic development in $T$. domestica. Also, our results indicate that $d s x$-like is not essential for the sexual differentiation of morphology during postembryonic development in T. domestica. The knockdown of both $d s x$ and $d s x$-like showed only the same effect as $d s x$ RNAi alone. Thus, it is unlikely that $d s x$-like functions redundantly with $d s x$. Sexual morphology, e.g., reproductive systems and genital organs, formed during postembryonic development is controlled by $d s x$ in males but is $d s x$ - 
324

\section{5}

326

327

independent in females of non-holometabolan insects such as T. domestica

(Zygentoma: this study), Bl. germanica (Dictyoptera: Wexler et al. 2019) and the brown planthopper Nilaparvata lugens (Hemiptera: Zhuo et al. 2018). There could be the possibility of tissue-specific effects of $d s x$ found in some holometabolan females (e.g., Ledón-Rettig et al. 2017). However, this possibility would be unlikely at least in these species since $d s x$ was knocked down by systematic RNAi and was not reported to affect female morphology at this time. Based on these facts, we estimate that $d s x$ may not be essential for female differentiation of morphology at the common ancestor of Dicondylia, ensuring the hypothesis proposed by Wexler et al. (2019).

$$
\text { To elucidate the timing of the acquisition of the role of } d s x \text { in female }
$$
morphogenesis during postembryonic development, we must interpret the role of $d s x$ in Hymenoptera, the basal clade of Holometabola. Studies in the honeybee Apis mellifera showed through genome editing that $d s x$ controls female differentiation of the internal reproductive system under worker nutrition conditions (Roth et al. 2019). In the honeybee, sex differences in the gonads are established during embryogenesis (Lago et al. 2020). Thus, the male-like reproductive organ in $d s x$ mutant females in Roth et al. (2019) would show an effect during embryogenesis, not during postembryonic development. We cannot conclude whether $d s x$ is not essential for female morphogenesis in the honeybee, since the information on the roles of $d s x$ in sexual morphology is limited to gonads and heads of the worker females. However, given that $d s x$ does not affect heads in Ap. mellifera females (Roth et al. 2019), wings in the parasitoid wasp Nasonia vitripennis females (Wang et al. 2020), and sexual traits in At. rosae females (Mine et al. 2017, 2021), at this time, it is reasonable to infer that $d s x$ was not essential for female morphogenesis during postembryonic development in the common ancestor of Hymenoptera. This interpretation and the 


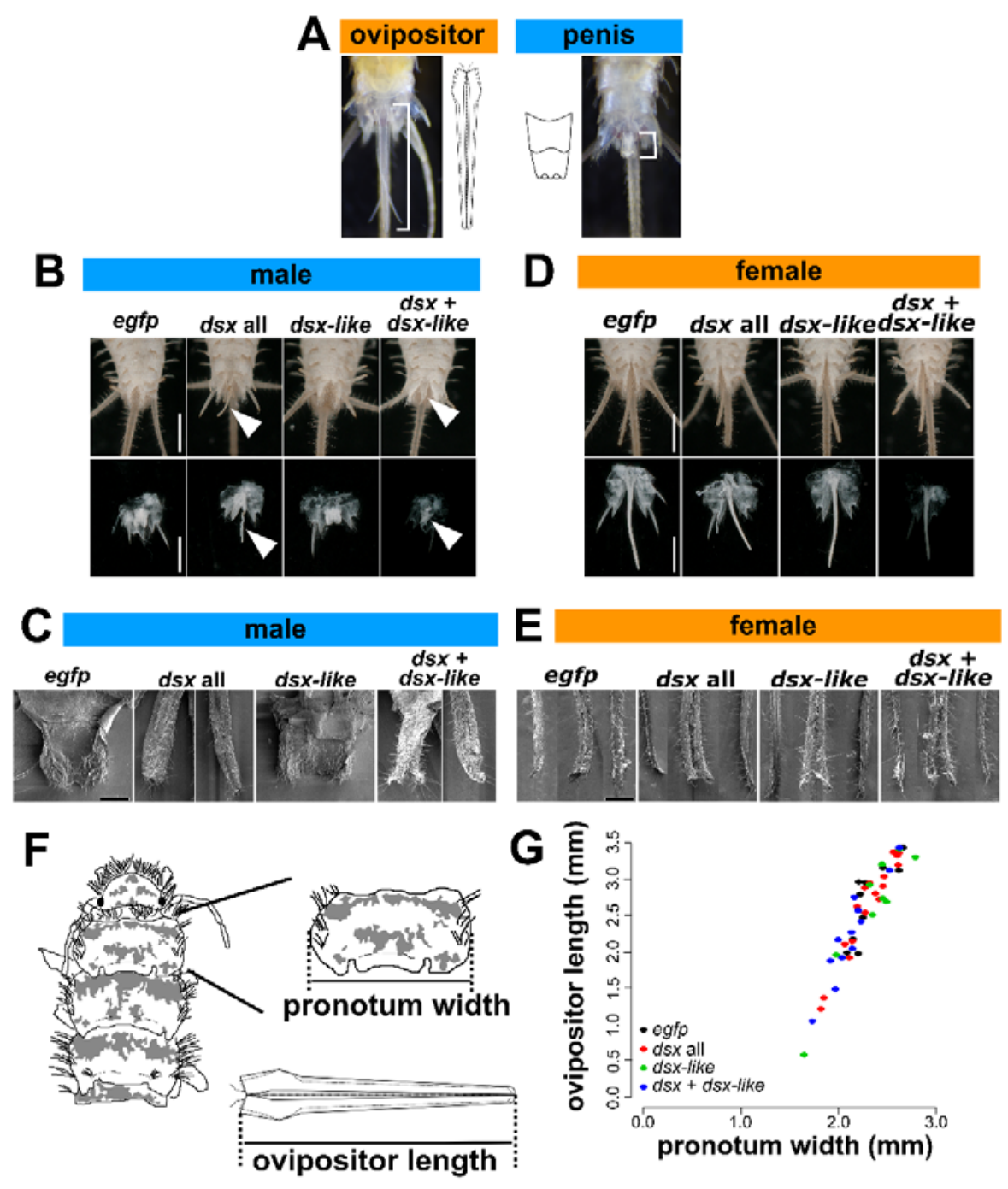

FIG. 3. Function of doublesex and doublesex-like for genital organs in Thermobia domestica. (A) Sexually dimorphic traits of $T$. domestica. Females possess an ovipositor and males have a penis. $(B)$ Effects of RNAi treatments on male penial structure. The upper images show the ventral side of the male abdomen. The lower images focus on the male penis. The arrowheads indicate the ovipositor-like structure in $d s x$ or both $d s x$ and $d s x$-like RNAi groups. (C) SEM images of male penial 
360

361

362

363

364

365

366

367

368

369

370

371

372

373

374

375

376

377

378

379

380

381

382

383

384

385

386

structure. In $d s x$ and $d s x+d s x$-like RNAi, the two photos are merged into the one image. In these images, the left panels show the ovipositor valvula II (inner sheath) like structure. The right panels exhibit the ovipositor valvula I (outer sheath)-like structure. The detail description can be referred in Supplementary Material online. $(D)$ Effects of RNAi treatments on female ovipositor. The upper images show the ventral side of the female abdomen. The lower images focus on the female ovipositor. $(E)$ SEM images of female ovipositor structure. In each image, the left and right panels show the valvula II and the middle one exhibits the valvula I. The results of the histological observation are in supplementary fig. 3 . The detail description can be referred in Supplementary material online. $(F)$ The schematic images of the measured parts. $(G)$ Effects of RNAi treatments on growth of ovipositor. Each plot indicates the ovipositor length of each individual. The results of the generalized linear model analysis show in supplementary table 4 . The ovipositor length was correlated with the prothoracic width $\left(P=2.00 \times 10^{-16}\right)$, although any significant effects can be seen in the RNAi treatments. Total $N=38$. In each panel, the egfp, $d s x$ all, $d s x$-like and $d s x+d s x$ like indicates the egfp dsRNA injected group (control), $d s x$ sex-common region dsRNA injected group, $d s x$-like dsRNA injected group, and both $d s x$ sex-common region and $d s x$-like dsRNAs injected group, respectively. Scales: $1 \mathrm{~cm}(B$ and $D) ; 50$ $\mu \mathrm{m}(C$ and $E)$.

\section{Cryptic role of doublesex for female-specific transcripts in T. domestica and its opposite role between sexes}

$d s x$ in $T$. domestica does not seem to have conflicting functions between sexes

in postembryonic morphogenesis. On the other hand, other biological processes

remain to be considered. We tested whether $d s x$ contributes to the expression of

vitellogenin (vtg), a yolk protein precursor gene that is highly expressed in animal

females (Byrne et al. 1989; Hayward et al. 2010). Previous studies have shown that

$v t g$ in pterygote insects is controlled by $d s x$ (e.g., Suzuki et al. 2003; Shukla and Palli

2012; Thongsaiklaing et al. 2018). Our RNA-seq analysis showed that three $v t g$

homologs, i.e., vtg $1, v \operatorname{tg} 2$, and $v \operatorname{tg} 3$, were expressed female-specifically in the fat body

in T. domestica (supplementary fig. 4; supplementary table 6). We analyzed the

expression of $v t g$ in the fat bodies of $d s x, d s x$-like, or both genes RNAi groups by RT-

qPCR.

In $d s x$ RNAi males, all $v t g$ mRNAs were expressed 45-1530-fold higher than the controls (fig. 4A; supplementary table 2: Brunner-Munzel test, $P=2.87 \times 10^{-8}$, 
395

396

397

$6.60 \times 10^{-16}$ and $2.80 \times 10^{-4}$ in $v \operatorname{tg} 1, v \operatorname{tg} 2$, and $\left.v \operatorname{tg} 3\right) . v \operatorname{tg} 1$ and $v \operatorname{tg} 3$ mRNAs were significantly up-regulated in $d s x$-like RNAi males compared to the controls (fig. 4A: Brunner-Munzel test, $P=0.0139$ and 0.00497 in $v \operatorname{tg} 1$ and $v \operatorname{tg} 3)$. In both $d s x$ and $d s x$ like RNAi males, the effect was similar to that in $d s x$ RNAi males (fig. 4A: BrunnerMunzel test, $P=6.60 \times 10^{-16}, 0.0162$, and $6.60 \times 10^{-16}$ in $v \operatorname{tg} 1$, vtg2, and $\left.v \operatorname{tg} 3\right)$. Then, we found that the expression of all $v t g$ genes was significantly reduced in $d s x$ RNAi females (fig. 4B; supplementary table 2: Brunner-Munzel test, $P=0.0433,0.00422$, and 0.00623 in $v \operatorname{tg} 1, v \operatorname{tg} 2$, and $v \operatorname{tg} 3)$. This reduction rate was approximately $0.2-0.4-$ fold. Furthermore, vtg expression was significantly reduced in $d s x$-like RNAi females (Brunner-Munzel test, $P=0.00256,3.80 \times 10^{-6}$, and $1.49 \times 10^{-5}$ in $v \operatorname{tg} 1, v \operatorname{tg} 2$, and $v \operatorname{tg} 3$ ) and both $d s x$ and $d s x$-like RNAi females (Brunner-Munzel test, $P=0.0305,0.00892$, and 0.0197 in $v t g 1, v t g 2$, and $v t g 3$ ) (fig. 4B). These results show that $d s x$ and $d s x$-like of $T$. domestica control $v t g$ negatively in males and positively in females.

Our results indicate that $d s x$ has opposite roles between sexes, i.e., repressive in males and promotive in females, in vtg expression. $d s x$-like also has the opposite functions for $v t g$ expression in males and females. It is unlikely that this result is due to $d s x$-like regulating $d s x$ transcription, as $d s x$-like did not affect $d s x$ expression (supplementary fig. 1A). A possible hypothesis is that $d s x$-like might regulate $v t g$ expression as one of the co-regulators that bind $d s x$ or other transcription factors. We do not know whether $d s x$ of $T$. domestica oppositely controls genes other than the $v t g$ homologs between sexes since our analysis was limited to $v t g$ homologs. However, the results from these genes indicates that the molecular function of $d s x$ in this species includes the opposite function for some genes' transcription in females and males. In Be.tabaci, $d s x$ positively regulates $v t g$ expression in females, even though it is not essential for female differentiation of morphological traits (Guo et al. 
420

421

422

423

424

425

426

427

428

429

430

431

432

433

434

435

436

437

438

439

440

441

442

443

444

2018). $d s x$ of this species does not negatively regulate $v t g$ in males. Therefore, the functionality of $d s x$ found in T. domestica, i.e., the opposing role in some genes' expression between sexes and the function that are not essential for female morphogenesis, is a functionality that has not been reported in any insect or animal. This functionality indicates that even if $d s x$ can oppositely function for some genes' expression between sexes, it does not necessarily have opposite functions in morphogenesis between sexes. This difference in the functionality might be due to differences in genes under $d s x$ control between morphogenesis and other aspects such as the yolk synthesis in females.

Genes under $d s x$ control in males are $d s x$-free in females of I. senegalensis (Takahashi et al. 2021), Bl. germanica (Wexler et al. 2019; Pei et al. 2021), and Ni. lugens (Zhuo et al. 2018). It was thought that feminizing roles of $d s x$ in morphogenesis and other biological processes may have appeared in the common ancestor of Aparaglossata (or Holometabola) as an entirely novel function, i.e., neofunctionalization. In contrast, the contribution of $d s x$ to some genes' expression in females of T. domestica (this study), Be. tabaci (Guo et al. 2018), Ap. mellifera (Velasque et al. 2018) and Aparaglossata raises the alternative hypothesis that the ability of $d s x$ to be involved in female differentiation was already present in the common ancestor of Dicondylia and later became essential for the female morphogenesis in the common ancestor of Aparaglossata. In this evolutionary scenario, the role of $d s x$ in the feminization of postembryonic morphogenesis in Aparaglossata could be due to extending its capability to control some genes in females, i.e., functional expansion. We cannot decide which of these hypotheses is appropriate, at this time. However, the latter scenario can well explain the presence of female-specific coding sequences of $d s x$ and high expression of $d s x$ female-type 


\section{A}

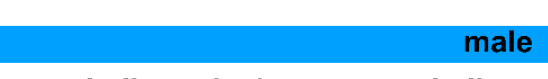

vitellogenin-1

vitellogenin-2

$* * * * * * * *$
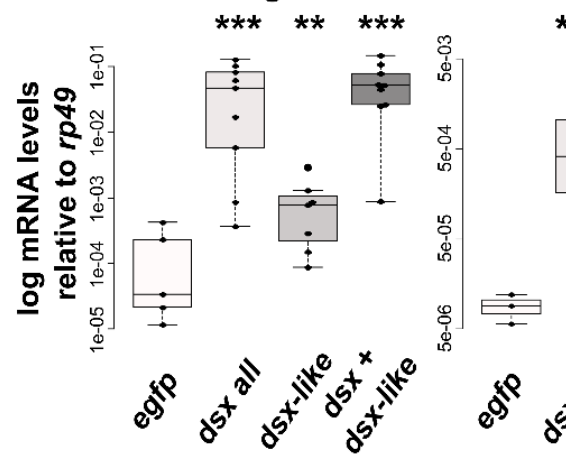

$\star \star \star$

***

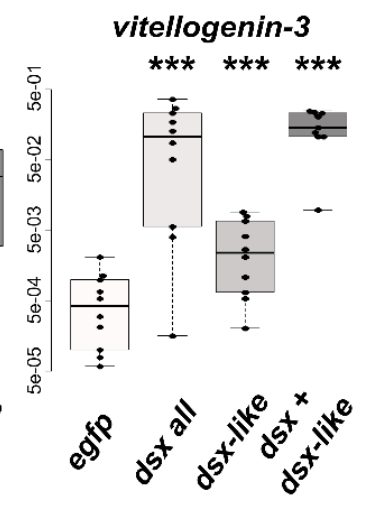

B
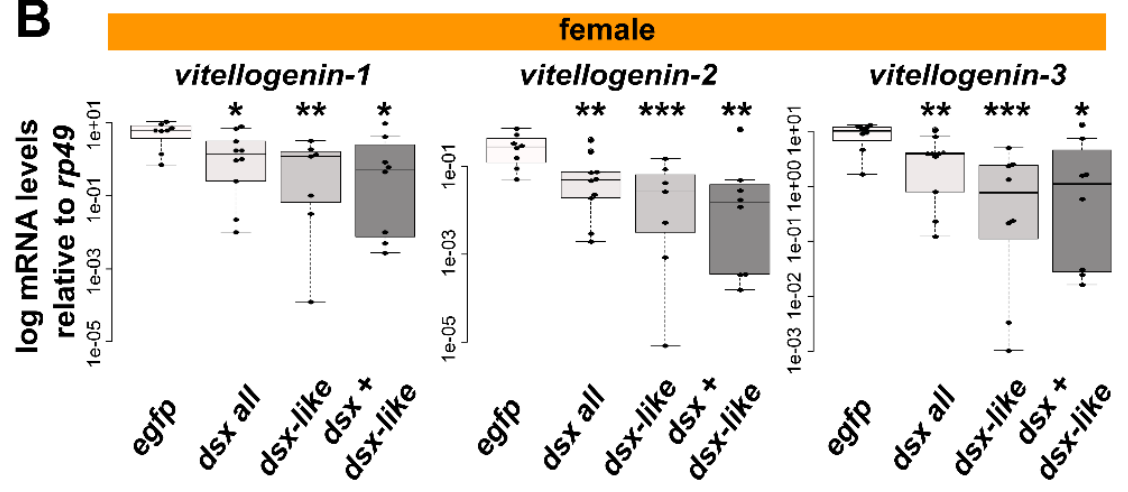

FIG. 4. Function of doublesex for vitellogenin expression in Thermobia domestica. (A) vitellogenin expression level in RNAi males. (B) vitellogenin expression level in RNAi females. The mRNA expression levels were measured by the RT-qPCR analysis. The figures show the log-scale relative values of the expression level of three vitellogenin homologs to the reference gene, ribosomal protein 49 (rp49). Each plot indicates the mRNA expression level of each individual. In each panel, the egfp, $d s x$ all, $d s x$-like and $d s x+d s x$-like indicates the egfp dsRNA injected group (control), $d s x$ sex-common region dsRNA injected group, $d s x$-like dsRNA injected group, and both $d s x$ sex-common region and $d s x$-like dsRNAs injected group, respectively. The Brunner-Munzel test method were performed to statistically analyze the difference in mRNA expression level between the control and the $d s x$ or $d s x$-like RNAi groups. The $P$-values were adjusted by the Holm's method. $* P<0.05, * * P<0.001, * * * P<0.0001 . P$ $\geq 0.05$ is not shown. The statistical results were described in supplementary table 2 . 
462

463

464

465

466

467

468

469

470

471

472

473

474

475

476

477

478

479

480

481

482

483

484

485

486

487

Total $N=30$ (vitellogenin-1), 24 (vitellogenin-2) and 39 (vitellogenin-3) in males and 33 (vitellogenin-1), 33 (vitellogenin-2), and 34 (vitellogenin-3) in females.

\section{Evolution of C-terminus disordered region of $d s x$ female-type}

One of the puzzling problems is how $d s x$ became recruited for female differentiation of morphological traits (cf., Hopkins and Kopp 2021). Here, we found that the C-terminal sequences including the oligomerization (OD) domain of the $d s x$ female-type is much shorter in $T$. domestica (38 aa) than that in D. melanogaster (53 aa) (supplementary fig. 5). The OD domain is essential for female differentiation in $D$. melanogaster, as it physically binds to $d s x$ itself, transcription factors, and coactivators (An and Wensink 1995; Erdman 1996; Ghosh et al. 2019; Romero-Pozuelo et al. 2019). Therefore, we hypothesized that the additive region found in $D$. melanogaster occurred at the common ancestor of Aparaglossata in which $d s x$ became essential for female morphogenesis. To test this hypothesis, we obtained sequences of $d s x$ female-type from 48 insect species based on the National Center for Biotechnology Information (NCBI) protein/transcriptome shotgun assembly database and previous studies (supplementary table 7) and reconstructed ancestral sequences of $d s x$ female-type. Our ancestral sequence reconstruction revealed that the C-terminal 16-amino acid region of $d s x$ female-type found in the common ancestor of Aparaglossata was absent in the common ancestor of the other taxon (fig. 5A; supplementary fig. 6; supplementary table 8). This motif is conserved within Aparaglossata in our dataset although moderate sequence diversification was observed (supplementary fig. 6). In our dataset, almost all sequences of this motif were not found in species in which $d s x$ is not essential for the female differentiation of morphological traits during postembryonic development. Exceptionally, $d s x$ of At. rosae had an amino acid sequence in the region corresponding to this motif, but our 
488

489

490

491

492

493

494

495

496

497

498

499

500

501

502

503

504

505

506

507

508

509

510

511

512

results of ancestral sequence reconstruction showed that this sequence was acquired in parallel with Aparaglossata.

The Aparaglossata-specific region is located in the distal (C-terminal) part of the female-specific region in D. melanogaster. This distal region is a disordered region, i.e., a mobile region that lacks a fixed structure, following an $\alpha$-helix loop in the proximal (N-terminal side) region (Yang et al. 2008). To investigate whether the acquisition of the disordered region occurred in Aparaglossata, we predicted the protein structure of $d s x$ female-type ancestral sequences of Pterygota, Neoptera, Eumetabola, Holometabola, and Aparaglossata. According to the Alphafold2 algorism-based structure prediction, the female-specific region of $d s x$ in the common ancestor of Aparaglossata had a proximal $\alpha$-helix loop structure, and a distal random coil indicating a disordered region (fig. 5B). This structure was similar to that of $D$. melanogaster determined by a crystal structural analysis (Yang et al. 2008). The proximal $\alpha$-helix loop structure was also predicted in the common ancestors of taxon other than Aparaglossata. The random coil following the $\alpha$-helix structure was predicted in all common ancestors, but its length was shorter than that of the common ancestor of Aparaglossata. It is essential to determine the structure via nuclear magnetic resonance or cryo-electron microscopy methods to conclude the details at the structural level, although, our theoretical predictions suggest that the disordered region following the $\alpha$-helix structure in the female-specific region may have been extended in the common ancestor of Aparaglossata.

Our results suggest that both the extension of the disordered region following the $\alpha$-helix loop in the female-specific region of $d s x$ and the feminizing function of $d s x$ for morphology occurred in the common ancestor of Aparaglossata. At present, the causality between these two events is uncertain, as we do not know which of the 
513 events appeared earlier. In general, disordered regions in transcription factors play

514 essential roles in transcriptional activity through post-translational modifications and

515 binding to co-activators and nucleic acids (Liu et al. 2006; Darling and Uversly 2018).

516 Furthermore, Wang et al. (2019) showed that in the diamondback moth Plutella

$517 x y$ lostella, when the Aparaglossata-specific motif is specifically broken by deletion or

518 frameshift mutations using the CRISPR/Cas9 method, the female morphology is

519 transformed into the intersexual phenotype. This result indicates that the

520 Aparaglossata-specific motif is essential for female differentiation of morphology in

521 P. xylostella. These facts suggest that the extension of the C-terminal region of $d s x$

522 female-type may have been a key event associated with the acquisition of the female-

523 differentiating roles of $d s x$ in morphology during postembryonic development. This

524 functional evolution of the "non-functional" isoform by the coding mutation is also

525 consistent with an evolutionary process of alternative splicing isoforms theoretically

526 predicted lacking empirical evidence (Keren et al. 2010). 

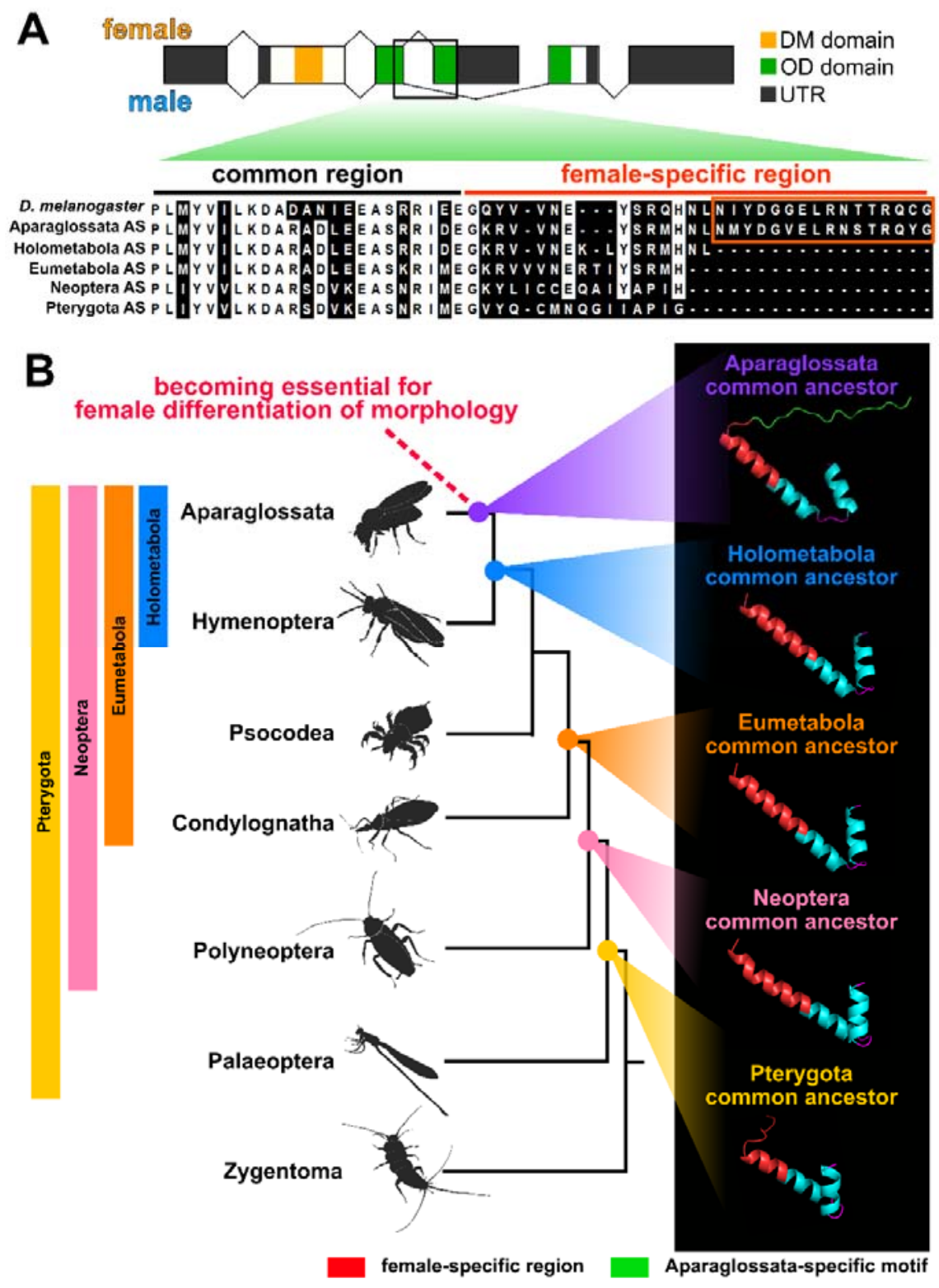

527 FIG. 5. Evolution of C-terminal sequence of doublesex in insects. (A) Ancestral sequences (AS) of $d s x$ in insects. The AS were reconstructed from $49 d s x$ proteins of insects by the maximum likelihood methods of the MEGA X. The information on the species and proteins used for the AS reconstruction is listed in supplementary table 7. The most probable sequences were applied. The results of the AS reconstruction are described in supplementary table 8 . The upper scheme indicates the $d s x$ gene structure of $D$. melanogaster. The lower image shows the result of the multiple sequence alignments (MSA) of $d s x$ sequences by MAFFT. The oligomerization domain sequences at C-terminal side were used for the MSA. The white background in the MSA result indicates the conserved sites that share the residues in the $80 \%$ taxa. The Aparaglossata-specific motif is indicated by the orange frame. $(B)$ Predicted protein structures of $d s x$ female-type in common ancestors of insect taxa. The phylogenetic 
539

540

541

542

543

544

545

546

547

548

549

550

551

552

553

554

555

556

557

558

559

560

561

562

563

564

565

566

relationship is based on the topology of Misof et al. (2014). The 3D images in the right panel indicate the predicted structures of the OD domain including the femalespecific region of $d s x$. The protein structures were predicted by the AlphaFold2-based algorism (ColabFold: Mirdita et al. 2021). The red region of the 3D image indicates the female-specific region. The green region shows the Aparaglossata-specific motif. The information on the evaluated values (predicted local distance difference test: pIDDT) of the prediction is shown in the Material and Methods section and supplementary fig. 10 .

\section{On the origin of outputs of the sexual differentiation mechanism}

Recent findings in insects (e.g., Mine et al. 2017; Guo et al. 2018; Zhuo et al.

2018; Wexler et al. 2019; Takahashi et al. 2021), including this study, have shown

that sexual differentiation mechanisms are diverse in their outputs as well as their

gene repertoires. The diversity in the output is attributed to the functional diversity of

a single gene, $d s x$, for sexual differentiation of morphogenesis during postembryonic

development. The evolutionary origin of the diversity in the output is one of the

enigmatic problems in sexual development. Information on the roles of $d s x$ is limited

to some traits in some species and cannot be available in many non-aparaglossatan

species although functional analyses of $d s x$ have been rapidly progressing using

emerging model species. Unquestionably, comprehensive information on functions of

$d s x$ for sexually dimorphic morphology from wider taxa is essential for fully tracing

the evolution of $d s x$. We propose, albeit premature, as one of the possibilities to be

considered, the hypothesis by which $d s x$ might have become essential for female

differentiation in sexual morphology by expanding its cryptic feminizing role, i.e.,

functions for some female genes' expression, in association with mutations in female-

specific motifs (fig. 6). This scenario can explain how single genes acquire novel

outputs of sexual development although our hypothesis does not prevent any other

alternative hypothesis from being proposed. 
567 The diversity of mechanisms that produce animal sex is a model case of

568 developmental systemic drift (True and Haag 2001; Haag and True 2021). The

569 functional diversity of a single gene and its evolutionary process has not been focused

570 on in the context of developmental system drift to date due to its poor examples. Our

571 evolutionary scenario may be one hypothesis explaining the origin of the system drift

572 in the function of single genes. In this study, we have mainly discussed the

573 functionality of $d s x$ for sexual differentiation of morphology during postembryonic

574 development. Therefore, it is unclear whether our conclusions and evolutionary

575 scenarios apply to sexual behavior, including sex pheromone secretion and courtship,

576 as well as sexual determination and gonadal differentiation during embryogenesis.

577 Detailed studies of sex differences at various levels across insect taxa will test our

578 evolutionary scenario and will fully reconstruct the evolutionary history of $d s x$ and

579 sexual differentiation mechanisms. 


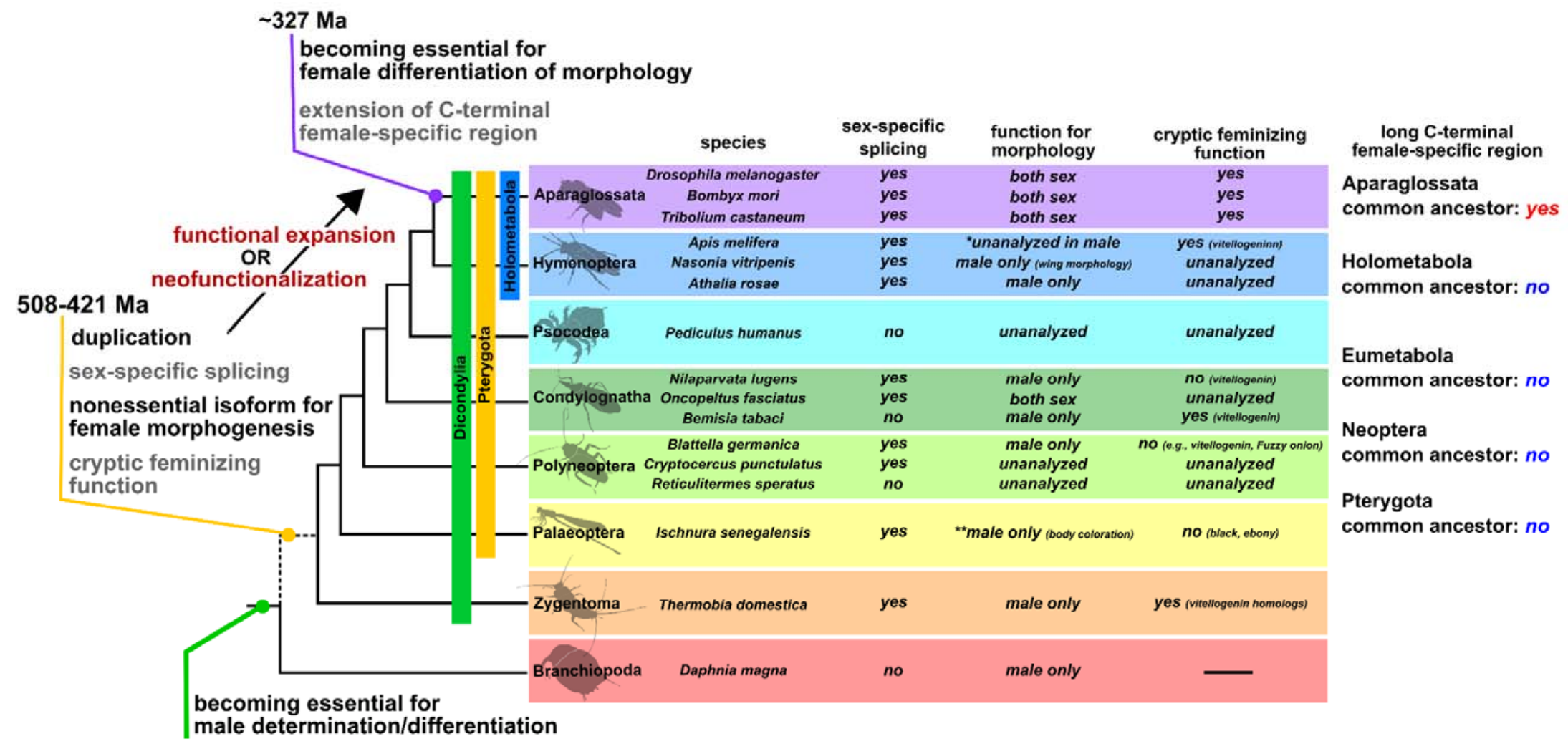

FIG. 6. Schematic image of the evolutionary scenario of doublesex proposed in this study and the feature of $d s x$ in insects. $d s x$ in arthropods may have been initially involved in only male determination/differentiation based on findings in crustaceans and chelicerates (Kato et al. 2011; Pomerantz et al. 2015; Li et al. 2018; Panara et al. 2019). In our hypothesis, the female isoform of $d s x$ may have been not essential for female differentiation of morphological traits at least from the common ancestor of Dicondylia to the common ancestor of Aparaglossata but might have contributed to the expression of some genes in females ("cryptic feminizing function" in the figure). This "seemingly non-functional" isoform might have become essential for female differentiation of morphological traits at the common ancestor of Aparaglossata emerging at $~ 327$ 
million years ago (Ma) through extending its cryptic feminizing function ("functional expansion" in the figure) or, alternatively, through acquiring entirely novel function ("neofunctionalization" in the figure). The extension of the C-terminus amino acid sequences in the femalespecific region might be involved in the functional expansion/neofunctionalization of $d s x$. The common ancestor between Branchiopoda and Hexapoda may have had the male-specific expressed $d s x$. It is estimated that the sex-specific splicing control and the gene duplication of $d s x$ occurred from the common ancestor between Branchiopoda and Hexapoda emerging at $\sim 508$ Ma to the common ancestor of Dicondylia at $\sim 421$ Ma. The phylogenetic relationship and the divergence time refer to Misof et al. (2014). The dotted line in the phylogenetic relationship indicates that the taxa that occurred from the common ancestor between Branchiopoda and Dicondylia to the common ancestor of Dicondylia are omitted. Here, we also show information on the current knowledge of $d s x$ features in insects and a branchiopod. In Aparaglossata, since there are many studies, we show only three representative species. The information was based on: Hildreth (1965), Bruce and Baker (1989) and Cloudh et al. (2014) in Drosophila melanogaster (Diptera), Ohbayashi et al. (2001), Suzuki et al. (2003), and Xu et al. (2017) in Bombyx mori (Lepidoptera), Shukla and Palli (2012) in Tribolium castaneum (Coleoptera), Roth et al. (2019) and Velasque et al. (2018) in Apis mellifera (Hymenoptera), Wang et al. (2020) in Nasonia vitripenis (Hymenoptera), Mine et al. (2017, 2021) in Athalia rosae (Hymenoptera), Wexler et al. (2019) in Pediculus humanus (Psocodea) and Blattella germanica (Dictyoptera), Zhuo et al. (2018) in Nilaparvata lugens (Hemiptera), Just et al. (2021) in Oncopeltus fasciatus (Hemiptera), Guo et al. (2018) in Bemisia tabaci (Hemiptera), Miyazaki et al. (2021) in the wood roach Cryptocercus punctulatus and Reticulitermes speratus (Dictyoptera), Takahashi et al. $(2019,2021)$ in Ischnura senegalensis (Odonata), this study in Thermobia domestica (Zygentoma), and Kato et al. (2011) in Daphnia magna (Branchiopoda). In Condylognatha, information on dsx in the blood-sucking bug Rhodnius prolixus is omitted. R. prolixus has sex-specific isoforms of $d s x$ whose function has not been investigated (Wexler et al. 2014). The "unanalyzed" means the functional analyses of $d s x$ have not been performed in the relevant species. Information on the roles of $d s x$ of some species in female morphogenesis is limited to some body parts: e.g., body coloration in I. senegalensis (Takahashi et al. 2021), wing morphology in Na. vitripenis (Wang et al. 2020), and worker morphology in Ap. mellifera (Roth et al. 2019). The asterisk (*) in Ap. mellifera indicates that the functional analysis of $d s x$ in males was not conducted although the gonad differentiation of female workers was affected by $d s x$ knockouts (Roth et al. 2019; see Main text). The double-asterisk (**) in I. senegalensis shows that this species has polymorphic coloration in females, i.e., gynomorph (normal female color) and andromorph (male-like color) and that $d s x$ is involved in the color formation of the males and andromorphic females but not gynomorphic females (see Takahashi et al. 2021), suggesting that $d s x$ is not essential for the female color development. In our hypothesis, the essential roles of $d s x$ for female development in $O$. fasciatus (Just et al. 2021) may have occurred in parallel with Aparaglossata. 


\section{Materials and Methods}

\section{Animals}

617 (Lepismatidae). The insects were kept at $37^{\circ} \mathrm{C}$ in total darkness condition and fed with

618 fish food (TetraFin Goldfish Flakes, Tetra GmbH, Melle, Germany) in our laboratory.

619 Stock colonies were reared in plastic cases of $30 \mathrm{~cm} \times 40 \mathrm{~cm}$ or $18 \mathrm{~cm} \times 25 \mathrm{~cm}$ in

620 length. Eggs were collected from tissue paper in the case and incubated at $37^{\circ} \mathrm{C}$. For

621 examining the roles of $d s x$ and $d s x$-like in the postembryonic morphogenesis, colonies

622 of hatched nymphs were reared up to the fourth instar in a six-well plate and then

623 transferred into 24-well plates to be kept individually. For examining the roles of $d s x$

624 and $d s x$-like in vitellogenin expression, female and male insects were collected from

625 the stock colony and transferred into the plates. For examining the function of $d s x$ and

$626 d s x$-like for sexual morphology and gametogenesis, we used firebrats from April to

627 June, 2019, February to April, April to July, and September to December, 2020. For

628 investigating the roles of $d s x$ and $d s x$-like in the vitellogenin expression, firebrats were

629 manipulated from June to July, 2020.

\section{Estimation of molt timing}

631 Estimating the molt timing of insects is essential for the analysis of

632 developmental processes and the functions of developmental regulatory genes. The

633 timing of Hemi- or holometabolan insects can be estimated using morphological

634 changes such as a wing growth. However, timing is hard to estimate in apterygote

635 insects since they have little change in their morphology during postembryonic

636 development. T. domestica forms scales in the fourth instar, and changes the number 
637 and length of its styli during the fourth to ninth instar under our breeding conditions.

638 These features can be used to estimate molt timing, but it is difficult to apply these

639 criteria to experiments using adults or a large number of nymphs. To resolve this

640 problem, we used leg regeneration after autotomy and time-lapse imaging to estimate

641 the molt timing of $T$. domestica. Autotomy occurs at the joint between the trochanter

642 and femur in T. domestica. An autotomized leg regenerates after one molt (Buck and

643 Edwards, 1990). For the RNAi analysis during postembryonic development, we

644 amputated a right hindleg at the autotomic rift, using tweezers, and observed whether

645 the leg had regenerated. This test enabled us to rapidly estimate the molt timing. For

646 the RNA-seq and the RT-qPCR analysis, the time-lapse imaging was used to

647 determine the precise time of molt. We build a time-lapse imaging system with a

648 network camera system (SANYO, Tokyo, Japan) set in an incubator at $37^{\circ} \mathrm{C}$

649 (supplementary fig. 7A). Photos of insects in the 24-well plate were taken every five

650 minutes. We created a time-lapse movie from the photos every 12 hours using Image J

651 1.52a (https://imagej.nih.gov/ij/) and observed whether the insects molted

652 (supplementary fig. 7B).

653 De novo genome assembly

654 A whole genome of T. domestica was sequenced to analyze the exon-intron

655 structure of $d s x$. We selected an adult female of $T$. domestica from our stock colony

656 and removed its alimentary canal. Genomic DNA was extracted from the sample

657 using DNeasy Blood and Tissue Kit (QIAGEN K.K., Tokyo, Japan). A paired-end

658 library was constructed from $1 \mu \mathrm{g}$ of the DNA using TruSeq DNA PCR-Free LT

659 Sample Prep kits (Illumina K.K., Tokyo, Japan) following the manufacturer's

660 instructions. The library was run on a sequencer (HiSeq 2500; Illumina K.K., Tokyo,

661 Japan). We obtained $417 \mathrm{~Gb}$ of raw reads and assembled them using Platanus v1.2.4 
662

663

664

665

666

667

668

669

670

671

672

673

674

675

676

677

678

679

680

681

682

683

684

685

686

assembler (Kajitani et al. 2014) after removal of the adapter sequences. The genome sequence can be obtained from the DNA Data Bank in Japan (Accession number:

DRA005797; Bioproject: PRJDB5781).

\section{Transcriptome analysis}

To search for doublesex ( $d s x)$ and vitellogenin (vtg) homologs, we performed RNA-seq analysis. Adults of 15 우 and $15 \hat{\delta} \widehat{\delta}$ of $T$. domestica were sampled 1440 minutes after a molt in December, 2019. The fat bodies of the individuals were removed using tweezers in a phosphated buffered saline (PBS; $\mathrm{pH}=7.2$ ). Three adults were used per sample. Total RNA was extracted from 10 samples $(5 ㅇ+q, 5 \hat{\jmath})$ using RNeasy Micro kits (QIAGEN K.K., Tokyo, Japan) following the manufacturer's instructions. The concentration of purified RNA was measured using a Qubit 4 fluorometer (QIAGEN K.K., Tokyo, Japan) with Qubit RNA BR Assay kits (QIAGEN K.K., Tokyo, Japan). Paired-end libraries were constructed from $100 \mathrm{ng}$ of the total RNAs using TruSeq RNA Library Prep kits v2 (Illumina K.K., Tokyo, Japan) following the manufacturer's instructions. The libraries were run on a sequence (Hiseq, Illumina, Tokyo, Japan). The library preparation and sequencing were performed by Genewiz Strand-Specific RNA-seq service. We mapped the reads obtained to the assembled genome using the HISAT2 program (Kim et al. 2019) with a default option and counted the mapped reads using the STRINGTie program (Pertea, 2015) with default parameter settings. Differential expression gene analysis was performed based on the count matrix using the "edgeR" package (Robinson et al. 2010) in R-v4.0.3 (R Core Team, 2020). Information about the samples can be obtained from the National Center for Biotechnology Information (NCBI) BioSample database (Accession number: SAMN18175012-SAMN18175021).

\section{Molecular phylogenetic analysis}


MAFFT version 7 (Katoh et al. 2013) with the -linsi option (to use an accuracy 2018). Bootstrap values were calculated after 1000 replications.

\section{Full-length cDNA and exon-intron structures}

We extracted total RNA from eggs, whole bodies, fat body, and gonads of nymphs

709 Center Inc., Ohio, USA) following the manufacturer's instructions. The total RNAs 
712

713

714

715

716

717

718

719

720

721

722

723

724

725

726

727

728

729

730

731

732

733

734

735

736

extraction and ethanol precipitation. For $5 \square$-RACE analysis, mRNAs were purified from $75 \mu \mathrm{g}$ of the total RNAs using Dynabeads mRNA Purification kit (Thermo Fisher Scientific K.K., Tokyo, Japan) following the manufacturer's instruction. We then ligated an RNA oligo at the 5'-end of the mRNA using GeneRacer Advanced

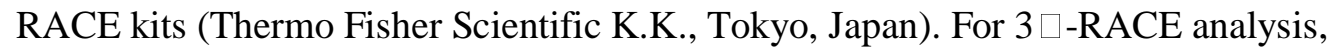
we ligated an RNA oligo of the SMART RACE cDNA Amplification Kit (Takara Bio Inc., Shiga, Japan) at $3 \square$-end of the total RNA during reverse transcription. First stranded (fs-) cDNA was generated from the RNAs using SuperScript III Reverse Transcriptase (Thermo Fisher Scientific K.K., Tokyo, Japan). We used primers specific to the RNA oligos and performed RACE analysis by nested RT-PCR using Q5 High-Fidelity DNA polymerase (New England BioLabs Japan Inc., Tokyo, Japan). The primers specific to $d s x$ and $d s x$-like were made from sequences of the relevant genomic regions and are listed in supplementary table 9. The amplicons were separated using the agarose gel-electrophoresis and cloned using TOPO TA Cloning Kit for Sequencing (Thermo Fisher Scientific K.K., Tokyo, Japan) following the manufacture's protocol. We used a DH5a Escherichia coli strain (TOYOBO CO., LTD., Osaka, Japan) as the host cell. Plasmids were extracted using the alkaline lysis and purified by phenol-chloroform and ethanol precipitation. The nucleotide sequences of the cloned amplicons were determined from the purified plasmids by the Sanger Sequencing service of FASMAC Co. Ltd. (Kanagawa, Japan). We then searched the genomic region of the full-length cDNA sequences of $d s x$ and $d s x$-like via local blastn analysis.

\section{Reverse transcription-quantitative PCR (RT-qPCR)}

To quantitative mRNA expression levels, we performed RT-qPCR analysis.

For investigating the sex-specific expression profile of $d s x$ and $d s x$-like, we used the 
737 fat body of adults of $T$. domestica since the sexes can be distinguishable by the

738 external morphology at this stage. Fat bodies also exhibit sex-specific physiological

739 functions in adults. Thirteenth instar individuals and adults after molting were

740 sampled for investigating roles of the genes in the sexually dimorphic morphology

741 and the vitellogenin expression, respectively. The sample sizes are reported in the

742 figure legends and supplementary table 2. We dissected the individuals in PBS and

743 collected their fat body in $2 \mathrm{ml}$ tubes containing TRI Reagent (Molecular Research

744 Center Inc., Ohio, USA). The fat bodies then were disrupted using a TissueLyser LT

745 small beads mill (QIAGEN K.K., Tokyo, Japan). These disrupted samples were

746 preserved at $-80^{\circ} \mathrm{C}$ until used. Total RNA was extracted from the samples according

747 to the manufacture's protocol for the TRI Reagent. Extracted RNA was treated with

$748 \quad 2 \%$ RNase-free DNase I (New England BioLabs Japan Inc., Tokyo, Japan) at $37^{\circ} \mathrm{C}$ for

74940 minutes and purified by phenol/chloroform extraction and ethanol precipitation.

750 We measured the concentration of the total RNA using a spectrophotometer (DS-11+,

751 Denovix Inc., Wilmington, USA). fs-cDNA was synthesized from $350 \mathrm{ng}$ of the total

752 RNA using SuperScript III Reverse Transcriptase (Thermo Fisher Scientific K.K.,

753 Tokyo, Japan). We diluted the fs-cDNA to 1:2 with MilliQ water and preserved it at

$754-30^{\circ} \mathrm{C}$ until it was used in RT-qPCR assay. The RT-qPCR assays were performed

755 using a LightCycler 96 instrument (Roche, Basel, Switzerland) according to the

756 manufacture's protocol with the THUNDERBIRD SYBR qPCR Mix (TOYOBO Co.

757 Ltd., Osaka, Japan). The reaction volume was $10 \mu \mathrm{l}$. We used $1 \mu 1$ of the fs-cDNA as

758 templates. The preparation of the RT-qPCR solution proceeded on ice. The protocol

759 of the RT-qPCR was as follows: preincubation at $95^{\circ} \mathrm{C}$ for 600 seconds and 45 cycles

760 of three-step reactions, such as denaturation at $95^{\circ} \mathrm{C}$ for 15 seconds, annealing at $60^{\circ} \mathrm{C}$

761 for 15 seconds and extension at $72^{\circ} \mathrm{C}$ for 45 seconds. We used ribosomal protein 49 
762 (rp49) as a reference gene, as described by Ohde et al. (2011). We designed primer

763 sets of the target genes by the Primer3Web version 4.1.0 (Untergasser et al. 2012)

764 following the manufacture's recommended condition of the THUNDERBIRD SYBR

765 qPCR Mix. We confirmed the primers'specificity using melting curves ranging from

$766 \quad 65^{\circ} \mathrm{C}$ to $95^{\circ} \mathrm{C}$. We selected primer sets exhibiting a single peak. The primers are listed

767 in supplementary table 9. Each RT-qPCR was technically replicated three times.

768 Some samples were excluded before analyzing the data when the Ct value of any

769 genes was not detected in one or more replicates or when the $\mathrm{Ct}$ value of the reference

770 gene deviated from that of other samples. In these removed data, a technical error was

771 suspected. We calculated the expression level of target genes by the $2^{-\Delta \Delta \mathrm{Ct}}$ method

772 (Livak and Schmittgen 2001) and performed the Brunner-Munzel (BM) test for $\Delta \mathrm{Ct}$

773 value. The BM test was carried out using R-v4.0.3. with the brunnermuzel.test

774 function of the "brunnermuzel" package (https://cran.r-

775 project.org/web/packages/brunnermunzel/index.html). Holm's method was used for

776 multiple comparison analyses between the control and treatments. The data are listed

777 in supplementary table 2 . In the $d s x$ expression of the RNAi male, we performed the

778 Smirnov-Grubbs (SG) test for $\Delta \mathrm{Ct}$ value using the grubbs.test function of the

779 “outliers” package in R (https://cran.r-project.org/web/packages/outliers/index.html)

780 (supplementary table 3). An outlier was detected in the $d s x$ RNAi male. We

781 repeatedly performed the SG test using the data excluding the outlier. No further

782 outliers were detected. Lastly, we re-analyzed the data, excluding the outlier, using

783 the BM test (supplementary table 2).

\section{RNAi analysis}

The RNAi assay can be used to examine the roles of genes during 
787

788

differentiation of insects is generally assumed to be a cell-autonomous mechanism that is independent of systemic hormonal- control (Verhulst and van de Zande 2015) as discussed in De Loof and Huybrechts (1998) and Bear and Monteiro (2013) and progresses during postembryonic development. Therefore, nymphal RNAi is the most effective tool to investigate the roles of genes on sexual trait formation during postembryonic development. To reduce the risk of off-target effects, the dsRNA was designed to avoid the region of the DM domain. We also confirmed that the dsRNA had no contiguous matches of more than 20 bases with other genes on the genome by BLAST (blastn option). To produce templates for the dsRNA, we cloned the regions of $d s x$ and $d s x$-like from the fs-cDNA using the same method as the RACE analysis. We amplified the template DNAs from purified plasmids with PCR using Q5 HighFidelity DNA Polymerase and purified the amplified DNA with the phenol/chloroform extraction and the ethanol precipitation. dsRNA was synthesized from the purified DNA using Ampliscribe T7-Flash Transcription kits (Epicentre Technologies, Co., Wisconsin, USA). We designed the PCR primers using the Primer3Web version 4.1.0 (Untergasser et al. 2012). The PCR primers are listed in supplementary table 9. In nymphal RNAi analysis, we injected the dsRNAs repeatedly into the abdomen of the nymphs of $T$. domestica with each molt from the fourth or fifth instar to thirteenth instar to sustain the RNAi effect during postembryonic development. The initial stage was the same within a single experiment. This repeated RNAi treatment was effective in some insects such as Blattella germanica (Wexler et al. 2019). We sampled the individuals one, three, and five days after molting, using phenotypic observations, analysis of $d s x$ knockdown effects, and the oocyte number.

To determine the sex of individuals, we initially observed the gonads: testis and ovary. In our RNAi analysis, the gonads completely formed and there was no 
812 difference between the control and $d s x$ RNAi individuals in external morphology (fig.

$8132 \mathrm{2}$ ). Therefore, individuals with testis were males and those with ovaries were

814 females. T. domestica molts throughout its life, even after sexual maturation, and

815 produces $v t g$ during each adult instar (Rousset and Bitsch 1993). To analyze the $v t g$

816 mRNA levels, we also injected the dsRNAs of $d s x$ and $d s x$-like repeatedly into the

817 females and males every three days from 12 hours after molting. We sampled the

818 females and males at $720 \pm 20$ minutes after subsequently molts.

\section{Phenotype observation}

820 We dissected thirteenth instar individuals in PBS using tweezers and removed

821 the thoraxes, reproductive systems, and external genital organs. We took images using

822 the digital microscope system (VHX-5000, KEYENCE, Tokyo, Japan). The thoraxes

823 and external genital organs were fixed with FAA fixative (formaldehyde: ethanol:

824 acetic acid $=15: 5: 1)$ at $25^{\circ} \mathrm{C}$ overnight and then preserved in $90 \%$ ethanol. We used

825 the length of the prothorax as an indicator of body size. To measure the prothoracic

826 width, the prothoracic notum was removed from the fixed thorax after treatment with

$82710 \% \mathrm{NaOH}$ solution at $60^{\circ} \mathrm{C}$ for 30 minutes to dissolve the soft tissues. The notum

828 was mounted in Lemosol on a microscope slide. The prepared specimens were imaged

829 using a KEYENCE VHX-5000. With the microscope at 50×, the length of the notum

830 was measured. The ovipositor length was also measured using the microscope at $20 x$

831 and 50x. To count the sperm number, sperm was collected from seminal vesicles and

832 diluted with $5 \mathrm{ml}$ MilliQ water. $50 \mu \mathrm{l}$ of the diluted sperm was spotted on a

833 microscope slide and dried overnight. We technically replicated the measurement

834 three times for ovipositor length and six times in sperm number and calculated these

835 means. Measurement was performed by blinding the treatment. We counted the

836 number of oocytes in ovarioles using an optical microscope at 50× (Olympus, Tokyo, 
837 Japan). A generalized linear model (GLM) was used to analyze differences in

838 ovipositor length (length data) and sperm and oocyte number (count data) among

839 RNAi treatments. The body size, target genes, and interactions between the target

840 genes were used as explanatory variables. The length was assumed to follow a

841 Gaussian distribution, and the count data to have a negative binomial distribution. We

842 used R-v4.0.3 in these analyses and the $g l m$ and the glm.nb (MASS package)

843 functions for the length and count data, respectively. To analyze the contribution of

844 the explanatory variables, a likelihood ratio test for the result of GLM was performed

845 using the Anova function of the car package. The statistical results are listed in

846 supplementary table 4 (female) and 5 (male).

\section{Scanning Electron Microscopy (SEM)}

848 The NanoSuit method (Takaku et al. 2013) was used for the SEM analysis.

849 Male penises and female ovipositors preserved in $90 \%$ ethanol were washed with

850 distilled water and immersed in $1 \%$ Tween 20 at $25^{\circ} \mathrm{C}$ for 10 minutes. The samples

851 were mounted on stubs and imaged using a low-vacuum SEM (DX-500; KEYENCE,

852 Tokyo, Japan).

853 Histology

$854 \quad$ The gonads of RNAi individuals were fixed with Bouin's fixative (saturated

855 picric acid: formaldehyde: glacial acetic acid $=15: 5: 1)$ at $25^{\circ} \mathrm{C}$ overnight and washed

856 with $90 \%$ ethanol plus Lithium Carbonate $\left(\mathrm{Li}_{2} \mathrm{CO}_{3}\right)$. The ovipositors of RNAi

857 individuals were fixed with FAA fixative at $25^{\circ} \mathrm{C}$ overnight and then were transferred

858 into $90 \%$ ethanol. The samples were dehydrated and cleared with an ethanol-butanol

859 series. The cleared samples were immersed and embedded in paraffin at $60^{\circ} \mathrm{C}$. The

860 paraffin blocks were polymerized at $4{ }^{\circ} \mathrm{C}$ and cut into $5 \mu \mathrm{m}$ thick sections using a

861 microtome (RM2155: Leica, Wetzlar, Germany). The sections were mounted on 
862 microscope slides coated with egg white-glycerin and stained using Delafield's

863 Hematoxylin and Eosin staining. After staining with the hematoxylin, the slides were

864 washed with $1 \%$ hydrochloric acid-ethanol for 40 seconds. The stained slides were

865 enclosed with Canada balsam. We observed the slides on an optical microscope

866 (Olympus, Tokyo, Japan) and took photos using a digital single-lens reflex camera

867 (Nikon, Tokyo, Japan).

868 Ancestral Sequence Reconstruction

869 To infer the sequence evolution of the $d s x$, we conducted an ancestral

870 sequence reconstruction (ASR) of the C-terminal sequences of the $d s x$ female-type

871 homologous sequence. First, we searched homologous sequences to $d s x$ female-type

872 from NCBI protein/transcript shotgun assembly databases and previous studies. The

873 searches in the NCBI databases were performed by BLAST search. We closely

874 examined the alignment results of the BLAST and selected sequences with at least 10

875 amino acids aligned with the female-specific region of each query sequence. We do

876 not know whether some of these sequences are expressed in females and contribute to

877 female morphogenesis, as these sequences are not necessarily to have investigated

878 expression and function in the species. We decided that it was not problem to use

879 these sequences since we focused on the evolution of sequences homologous to $d s x$

880 female-type in each insect taxa. In Diptera, we set $d s x$ female-type of $D$. melanogaster

881 (Accession \#: NP_001287220) as a query and obtained 9 sequences. In Lepidoptera,

882 we used $d s x$ female-type of B. mori (NP_001036871) as a query and get 10 sequences.

883 In Coleoptera, $d s x$ female-type of Tribolium castaneum (AFQ62106) was set in a

884 query and then 10 sequences were obtained. We used $d s x$ female-type of Ap. mellifera

885 (NP_001128407) and At. rosae (XP_012262256) as queries to search hymenopteran

886 sequences. We also searched some hymenopteran sequences from the NCBI databases 
887 based on a previous study (Baral et al. 2019). 10 hymenopteran sequences were

888 obtained. In Psocodea and Hymenoptera, we searched the databases to set the

889 sequences of Pediculus humanus (QGB21102) and Rhodonius prolixus (QGB21099)

890 as queries. Wexler et al. (2019) showed that $d s x$ of Pediculus humanus (Psocodea) has

891 isoforms without sex-specificity. In this study, based on the blast search and exon

892 structure, we regarded that the PhDsx1 in Wexler et al. (2019) is homologous to the

$893 d s x$ female-type. The sequences of Ni. lugens (AWJ25056) and Bl. germanica

894 (QGB21105 and QGB21106) were obtained from the database based on previous

895 studies (Zhuo et al. 2018; Wexler et al. 2019). We selected two sequences from $B l$.

896 germanica, as this species has two female-specific $d s x$ isoforms (Wexler et al. 2019).

897 The sequences of Cryptocercus punctulatus and I. senegalensis were obtained from

898 previous studies (Miyazaki et al. 2021; Takahashi et al. 2021). In T. domestica, the

899 sequence identified in this study was used. The sequence names are listed in

900 supplementary table 7 . We then manually extracted the OD domain and performed

901 multiple sequence alignments (MSA) using the MAFFT version 7 (Katoh et al. 2013)

902 with the -linsi option (to use an accuracy option, L-INS-i) (supplementary sequence

903 file 2). We reconstructed ancestral sequences (AS) from the MSA using MEGA X

904 software. The maximum-likelihood method was applied to the ASR. The JTT + G

905 model was chosen as a substitution model by AIC-based model selection. The guide

906 tree was reconstructed based on previously reported phylogenetic relationships

907 (Wiegmann et al. 2011; Misof et al. 2014; Li et al. 2017; Peters et al. 2017; Zhang et

908 al. 2018; Kawahara et al. 2019; McKenna et al. 2019; Gustafson et al. 2020)

909 (supplementary fig. 9). We selected the most probable sequences for the following

910 analyses. The results of ASR can be seen in supplementary table 8 . The probabilities

911 of sites of AS that we focused on are listed in supplementary table 10. In 
912 Aparaglossata (Node 77) and Holometabola (Node 87) AS, almost all probabilities of

913 sites were more than 0.9. The except sites were sites 83 and 98 in Node 77 and sites

$91477-79$ and 83 in Node 87 . These sites other than sites 77 had probabilities $>0.5$. Thus,

915 we concluded that the AS in Aparaglossata and Holometabola, which we considered

916 the most critical, was reconstructed with sufficient reliability. Any residues had the

917 probabilities $=0$ in the Aparaglossata-specific region of Holometabola AS. In

918 contrast, in non-holometabolan insects, since our taxon sampling is limited to several

919 species (Eumetabola in Node 92, Neoptera in Node95, Pterygota in Node 96), the

920 probabilities of some sites are lower than 0.5 . These low probable sites are not

921 necessarily confident. To conclude with reliability, it is no doubt that analyses based

922 on a larger number of species will be essential. However, all sites of the

923 Aparaglossata-specific region in these AS were gaps with the probabilities $>0.9$. The

924 result of the sites of the Aparaglossata-specific region seems to be relatively reliable

925 in our analysis. Thus, our conclusion that the Aparaglossata-specific region occurred

926 in the common ancestor of Aparaglossata would be confident. To compare the

927 sequences, we then performed MSA of the most probable reconstructed ancestral

928 sequences and the sequence of D. melanogaster using MAFFT version 7 (fig. 5A).

\section{Protein Structure Prediction}

930 To infer the evolution of protein structures of $d s x$, we conducted the protein

931 structure prediction. The ancestral sequences reconstructed by the above section were

932 used for the protein structure prediction. The sequences were obtained from

933 supplementary sequence file 3 . The protein structure prediction was performed using

934 the Alphafold2-based algorism (ColabFold: Mirdita et al. 2021) with the default

935 option. The accuracy of predictions was evaluated based on the predicted Local

936 distance difference test (plDDT) score that was automatically calculated on the 
937 ColabFold. We selected a model with the highest average plDDT score in each

938 prediction. The average plDDT scores were 81.824 (Aparaglossata), 89.165

939 (Holometabola), 87.376 (Eumetabola), 90.721 (Neoptera), and 90.720 (Pterygota).

940 The plDDT scores were more than 70 in the helical structure predicted as the $\alpha$-helix

941 loop of the female-specific $d s x$ region. Generally, predicted structures of plDDT $>70$

942 are regarded to be a confident prediction (cf., Tunyasuvunakool et al. 2021).

943 Therefore, we assessed the $\alpha$-helix loop of the female-specific region of $d s x$ as the

944 confidently predicted structure. The graph of the plDDT score of each model is shown

945 in supplementary fig. 10. The 3D models of predicted structures were visualized with

946 the PyMOL Molecular Graphics System, Version 2.0 (Schrödinger, LLC.). On the

947 viewer, we colored the female-specific region and the Aparaglossata-specific region

948 with red color and the green color, respectively.

\section{Data availability}

950 The draft genome data was deposited in the DNA Data Bank of Japan (Accession

951 number: DRA005797; Bioproject: PRJDB5781). The raw read data of the

952 transcriptome was in the NCBI Sequence Read Archive (Accession numbers:

953 SRR13870115-SRR13870124; Bioproject: PRJNA707122). The sequences of $d s x$

954 male-type, $d s x$ female-type, and $d s x$-like are also in GenBank (Accession numbers:

955 MW711323, MW711324, and MW711325, respectively).

956

\section{Acknowledgments}

958 We express gratitude to Dr. Daniel Bopp (University of Zürich) for his comments and

959 encouragement for this manuscript. We would like to thank Dr. Takahiro Ohde

960 (National Institute for Basic Biology; Kyoto University) for his help to extract

961 genomic DNA of T. domestica and for a great advice to the discussion. We are also 
962 grateful to Dr. Toshiya Ando, Dr. Taro Nakamura, Dr. Shinichi Morita, Dr. Hiroki

963 Sakai, and Dr. Tatsuro Konagaya (National Institute for Basic Biology) for technical

964 advice and discussion on this manuscript. We also express our gratitude to Dr. Satoshi

965 Miyazaki (Tamagawa university) for providing sequences of $d s x$ in Cryptocercus

966 punctulatus. Computations were performed on the NIG supercomputer at ROIS,

967 National Institute of Genetics and the Data Integration and Analysis Facility, National

968 Institute for Basic Biology. We thank the Model Plant Research Facility, NIBB

969 Bioresource Center for providing the network camera system. This work was

970 supported by the JSPS KAKENHI Grant numbers JP25660265, JP16H02596, and

971 JP16H06279 (PAGS) for TN and the Sasakawa Scientific Research Grant from The

972 Japan Science Society for YC.

973 Author Contributions:

974 YC and TN conceived this study. YC performed all experiments, observations, and

975 analyses other than the genome sequence and assembly. AT sequenced the genome.

$976 \mathrm{MO}$ and TI performed the de novo genome assembly. YC and TI wrote the

977 manuscript. All authors commented on the manuscript.

\section{Competing Interest Statement}

979 The authors declare that have no competing interests.

\section{References}

982 An W, Wensink PC. 1995. Integrating sex-and tissue-specific regulation within a

983 single Drosophila enhancer. Genes Dev. 9: 256-266. 
984

985

986

987

988

989

990

991

992

993

994

995

996

997

998

999

1000

1001

1002

1003

Bachtrog D, Mank JE, Peichel CL, Kirkpatrick M, Otto SP, Ashman TL, Hahn MW, Kitano J, Mayrose I, Ming R, et al. 2014. Sex determination: why so many ways of doing it? PLoS Biol. 12: e1001899.

Baral S, Arumugam G, Deshmukh R, Kunte K. 2019. Genetic architecture and sexspecific selection govern modular, male-biased evolution of doublesex. Sci Adv. 5: eaau3753.

Bear A, Monteiro A. 2013. Both cell-autonomous mechanisms and hormones contribute to sexual development in vertebrates and insects. Bioessays 35: 725-732.

Beukeboom LW, Perrin N. 2014. The evolution of sex determination. Oxford: Oxford University Press.

Beutel RG, Yavorskaya MI, Mashimo Y, Fukui M, Meusemann K. 2017. The phylogeny of Hexapoda (Arthropoda) and the evolution of megadiversity. Proc Arthropod Embryol Soc Jap. 51: 1-15.

Bopp D, Saccone G, Beye M. 2014. Sex determination in insects: variations on a common theme. Sex Dev. 8: 20-28.

Boudinot BE. 2018. A general theory of genital homologies for the Hexapoda (Pancrustacea) derived from skeletomuscular correspondences, with emphasis on the Endopterygota. Arthropod Struct Dev. 47: 563-613.

Buck C, Edwards JS. 1990. The effect of appendage and scale loss on instar duration in adult firebrats, Thermobia domestica (Thysanura). J Exp Biol. 151: 341-347. 
1004

1005

1006

1007

1008

1009

1010

1011

1012

1013

1014

1015

1016

1017

1018

1019

1020

1021

1022

1023

1024

Burtis KC, Baker BS. 1989. Drosophila doublesex gene controls somatic sexual differentiation by producing alternatively spliced mRNAs encoding related sexspecific polypeptides. Cell 56: $997-1010$.

Byrne BM, Gruber MABG, Ab G. 1989. The evolution of egg yolk proteins. Prog Biophys Mol Biol. 53: 33-69.

Clough E, Jimenez E, Kim YA, Whitworth C, Neville MC, Hempel LU, Pavlou HJ, Chen ZX, Sturgill D, Dale RK. 2014. Sex- and tissue-specific functions of Drosophila Doublesex transcription factor target genes. Dev Cell 31: 761-773.

Darling AL, Uversky VN. 2018. Intrinsic disorder and posttranslational modifications: the darker side of the biological dark matter. Front Genet. 9: 158.

Darwin C. 1871. The Descent of Man, and Selection in Relation to Sex vol. 1. London: John Murray.

De Loof A, Huybrechts R. 1998. "Insects do not have sex hormones": a myth? Gen Comp Endocrinol. 111: 245-260.

Emeljanov AF. 2014. The evolutionary role and fate of the primary ovipositor in insects. Entomol Rev. 94: 367-396.

Erdman SE, Chen HJ, Burtis KC. 1996. Functional and genetic characterization of the oligomerization and DNA binding properties of the Drosophila doublesex proteins. Genetics 144: 1639-1652.

Fryxell DC, Weiler DE, Kinnison MT, Palkovacs EP. 2019. Eco-evolutionary dynamics of sexual dimorphism. Trends Ecol Evol. 34: 591-594. 
1025

1026

1027

1028

1029

1030

1031

1032

1033

1034

1035

1036

1037

1038

1039

1040

1041

1042

1043

1044

1045

Geddes P, Thomson JA. 1889. The evolution of sex. London: Walter Scott.

Ghosh N, Bakshi A, Khandelwal R, Rajan SG, Joshi R. 2019. The Hox gene Abdominal-B uses Doublesex $\mathrm{x}^{\mathrm{F}}$ as a cofactor to promote neuroblast apoptosis in the Drosophila central nervous system. Development 146: dev175158.

Gotoh H, Zinna RA, Warren I, DeNieu M, Niimi T, Dworkin I, Emlen DJ, Miura T, Lavine LC. 2016. Identification and functional analyses of sex determination genes in the sexually dimorphic stag beetle Cyclommatus metallifer. BMC Genomics 17: 250.

Gubbay J, Collignon J, Koopman P, Capel B, Economou A, Münsterberg A, Vivian N, Goodfellow P, Lovell-Badge R. 1990. A gene mapping to the sex-determining region of the mouse $\mathrm{Y}$ chromosome is a member of a novel family of embryonically expressed genes. Nature 346: 245-250.

Guo L, Xie W, Liu Y, Yang Z, Yang X, Xia J, Wang S, Wu Q, Zhang Y. 2018. Identification and characterization of doublesex in Bemisia tabaci. Insect Mol Biol. 27: 602-632.

Gustafson GT, Baca SM, Alexander AM, Short AEZ. 2020. Phylogenomic analysis of the beetle suborder Adephaga with comparison of tailored and generalized ultraconserved element probe performance. Syst Entomol. 45: 552-570.

Haag ES, True JR. 2021. Developmental system drift. In: Nuño de la Rosa L, Müller GB, editors. Evolutionary developmental biology: a reference guide. Switzerland: Springer Nature Switzerland AG. P. 99-110. 
1046

1047

1048

1049

1050

1051

1052

1053

1054

1055

1056

1057

1058

1059

1060

1061

1062

1063

1064

1065

1066

1067

Hasselmann M, Gempe T, Schiøtt M, Nunes-Silva CG, Otte M, Beye M. 2008.

Evidence for the evolutionary nascence of a novel sex determination pathway in honeybees. Nature 454: 519-522.

Hattori RS, Murai Y, Oura M, Masuda S, Majhi SK, Sakamoto T, Fernandino JI, Somoza GM, Yokota M, Strüssmann CA. 2012. A Y-linked anti-Müllerian hormone duplication takes over a critical role in sex determination. Proc Natl Acad Sci USA. 109: 2955-2959.

Hayward A, Takahashi T, Bendena WG, Tobe SS, Hui JH. 2010. Comparative genomic and phylogenetic analysis of vitellogenin and other large lipid transfer proteins in metazoans. FEBS Letters 584: 1273-1278.

Herpin A, Schartl M. 2015. Plasticity of gene-regulatory networks controlling sex determination: of masters, slaves, usual suspects, newcomers, and usurpators. EMBO Reports 16: 1260-1274.

Hildreth PE. 1965. Doublesex, a recessive gene that transforms both males and females of Drosophila into intersexes. Genetics 51: 659-678.

Hildreth PE, Lucchesi JC. 1963. A gene which transforms males and females into intersexes. Proc 11th Intern Congr Genet. 1: 171.

Hopkins BR, Kopp A. 2021. Evolution of sexual development and sexual dimorphism in insects. Curr Opin Genet Dev. 69: 129-139.

Ito Y, Harigai A, Nakata M, Hosoya T, Araya K, Oba Y, Ito A, Ohde T, Yaginuma T, Niimi T. 2013. The role of doublesex in the evolution of exaggerated horns in the Japanese rhinoceros beetle. EMBO Reports 14: 561-567. 
1068

1069

1070

1071

1072

1073

1074

1075

1076

1077

1078

1079

1080

1081

1082

1083

1084

1085

1086

1087

1088
Just J, Laslo M, Lee YJ, Yarnell M, Zhang Z, Angelini DR. 2021. Distinct developmental mechanisms influence sexual dimorphisms in the milkweed bug Oncopeltus fasciatus. bioRxiv. doi: 10.1101/2021.05.12.443917.

Kajitani R, Toshimoto K, Noguchi H, Toyoda A, Ogura Y, Okuno M, Yabana M, Harada M, Nagayasu E, Maruyama H, Kohara Y, Fujiyama A, Hayashi T, Itoh T. 2014. Efficient de novo assembly of highly heterozygous genomes from wholegenome shotgun short reads. Genome Res. 24:1384-95.

Kato Y, Kobayashi K, Watanabe H, Iguchi T. 2011. Environmental sex determination in the branchiopod crustacean Daphnia magna: deep conservation of a Doublesex gene in the sex-determining pathway. PLOS Genet. 7: e1001345.

Katoh K, Standley DM. 2013. MAFFT multiple sequence alignment software version 7: improvements in performance and usability. Mol Biol Evol. 30: 772-780.

Kawahara AY, Plotkin D, Espeland M, Meusemann K, Toussaint EFA, Donath A, Gimnich F, Frandsen PB, Zwick A, dos Reis M, et al. 2019. Phylogenomics reveals the evolutionary timing and pattern of butterflies and moths. Proc Natl Acad Sci USA. 116: 22657-22663.

Keren H, Lev-Maor G, Ast G. 2010. Alternative splicing and evolution: diversification, exon definition and function. Nat Rev Genet. 11: 345-355.

Kijimoto T, Moczek AP, Andrews J. 2012. Diversification of doublesex function underlies morph-, sex-, and species-specific development of beetle horns. Proc Natl Acad Sci USA. 109: 20526-20531. 
1089

1090

1091

1092

1093

1094

1095

1096

1097

1098

1099

1100

1101

1102

1103

1104

1105

1106

1107

1108

1109

Kim D, Paggi JM, Park C, Bennett C, Salzberg SL. 2019. Graph-based genome alignment and genotyping with HISAT2 and HISAT-genotype. Nat Biotechnol. 37: 907-915.

Klag J. 1977. Differentiation of primordial germ cells in the embryonic development of Thermobia domestica, Pack. (Thysanura): an ultrastructural study. J Embryol exp Morph. 38: 93-114.

Koopman P, Gubbay J, Vivian N, Goodfellow P, Lovell-Badge R. 1991. Male development of chromosomally female mice transgenic for Sry. Nature 351: 117 121.

Kopp A. 2012. Dmrt genes in the development and evolution of sexual dimorphism. Trends Genet. 28: 175-184.

Kristensen NP. 1975. The phylogeny of hexapod "orders". A critical review of recent accounts. J Zool Syst Evol Res. 13: 1-44.

Kumar S, Stecher G, Li M, Knyaz C, Tamura K. 2018. MEGA X: molecular evolutionary genetics analysis across computing platforms. Mol Biol Evol. 35: $1547-1549$.

Lago DC, Martins JR, Dallacqua RP, Santos DE, Bitondi MM, Hartfelder K. 2020. Testis development and spermatogenesis in drones of the honey bee, Apis mellifera L. Apidologie 51: 935-955.

Li H, Leavengood JM, Chapman EG, Burkhardt D, Song F, Jiang P, Liu J, Zhou X, Cai W. 2017. Mitochondrial phylogenomics of Hemiptera reveals adaptive 

20171223. gene which regulates the expression of insulin-like androgenic gland hormone in Fenneropenaeus chinensis. Gene 649: 1-7. disorder in transcription factors. Biochemistry 45: 6873-6888.

Livak KJ, Schmittgen TD. 2001. Analysis of relative gene expression data using real-

Ledón-Rettig C, Zattara E, Moczek A. 2017. Asymmetric interactions between doublesex and tissue- and sex-specific target genes mediate sexual dimorphism in beetles. Nat Commun. 8: 14593.

Matsuda M, Nagahama Y, Shinomiya A, Sato T, Matsuda C, Kobayashi T, Morrey DMY is a Y-specific DM-domain gene required for male development in the medaka fish. Nature 417: 559-563.

Matsuda R.1976. Morphology and Evolution of the Insect Abdomen: With Special Reference to Developmental Patterns and their Bearings upon Systematics. Oxford:

Mawaribuchi S, Ito Y, Ito M. 2019. Independent evolution for sex determination and differentiation in the DMRT family in animals. Biol Open 8: bio041962. 
1131

1132

1133

1134

1135

1136

1137

1138

1139

1140

1141

1142

1143

1144

1145

1146

1147

1148

1149

1150

1151

McKenna DD, Shin S, Ahrens D, Balke M, Beza-Beza C, Clarke DJ, Donath A, Escalona HE, Friedrich F, Letsch H, et al. 2019. The evolution and genomic basis of beetle diversity. Proc. Natl. Acad. Sci. USA. 116: 24729-24737.

Mine S, Sumitani M, Aoki F, Hatakeyama M, Suzuki MG. 2017. Identification and functional characterization of the sex-determining gene doublesex in the sawfly, Athalia rosae (Hymenoptera: Tenthredinidae). Appl. Entomol. Zool. 52: 497-509.

Mine S, Sumitani M, Aoki F, Hatakeyama M, Suzuki MG. 2021. Effects of functional depletion of doublesex on male development in the sawfly, Athalia rosae. Insects, 12: 849 .

Mirdita M, Ovchinnikov S, Steinegger M. 2021. ColabFold-Making protein folding accessible to all. bioRxiv. doi: 10.1101/2021.08.15.456425.

Misof B, Liu S, Meusemann K, Peters RS, Donath A, Mayer C, Frandsen PB, Ware J, Flouri T, Beutel RG, et al. 2014. Phylogenomics resolves the timing and pattern of insect evolution. Science 346: 763-767.

Miyawaki S, Kuroki S, Maeda R, Okashita N, Koopman P, Tachibana M. 2020. The mouse Sry locus harbors a cryptic exon that is essential for male sex determination. Science 370: 121-124.

Miyazaki S, Fujiwara K, Kai K, Masuoka Y, Gotoh H, Niimi T, Hayashi Y, Shigenobu S, Maekawa K. 2021. Evolutionary transition of doublesex regulation in termites and cockroaches: from sex-specific splicing to male-specific transcription. Sci Rep. 11: 15992. 
1152 Morita S, Ando T, Maeno A, Mizutani T, Mase M, Shigenobu S, Niimi T. 2019.

1153 Precise staging of beetle horn formation in Trypoxylus dichotomus reveals the

1154 pleiotropic roles of doublesex depending on the spatiotemporal developmental

$1155 \quad$ contexts. PLOS Genet. 15: e1008063.

1156 Nanda I, Kondo M, Hornung U, Asakawa S, Winkler C, Shimizu A, Shan Z, Haaf T,

1157 Shimizu N, Shima A, Schmid M, Schartl M. 2002. A duplicated copy of DMRT1

1158 in the sex-determining region of the Y chromosome of the medaka, Oryzias latipes.

1159 Proc Natl Acad Sci USA. 99: 11778-11783.

1160 Ohbayashi F, Suzuki MG, Mita K, Okano K, Shimada T. 2001. A homologue of the

1161 Drosophila doublesex gene is transcribed into sex-specific mRNA isoforms in the

1162 silkworm, Bombyx mori. Comp Biochem Physiol B Biochem Mol Biol. 128: 145-

1163158.

1164 Ohde T, Yaginuma T, Niimi T. 2011. Nymphal RNAi analysis reveals novel function

1165 of scalloped in antenna, cercus and caudal filament formation in the firebrat,

1166 Thermobia domestica. J Insect Biotechnol Sericol. 80: 101-108.

1167 Panara V, Budd GE, Janssen R. 2019. Phylogenetic analysis and embryonic

1168 expression of panarthropod Dmrt genes. Front Zool. 16: 1-18.

1169 Pei XJ, Fan YL, Bai Y, Bai TT, Schal C,Zhang ZF, Chen N, Li S, Liu TX. 2021.

1170 Modulation of fatty acid elongation in cockroaches sustains sexually dimorphic

1171 hydrocarbons and female attractiveness. PLOS Biol. 19: e3001330. 
1172 Pertea M, Pertea GM, Antonescu CM, Chang TC, Mendell JT, Salzberg SL. 2015.

1173 StringTie enables improved reconstruction of a transcriptome from RNA-seq reads.

$1174 \quad$ Nat Biotechnol. 33: 290-295.

1175 Peters RS, Krogmann L, Mayer C, Donath A, Gunkel S, Meusemann K, Kozlov A,

1176 Podsiadlowski L, Petersen M, Lanfear R, et al. 2017. Evolutionary History of the

1177 Hymenoptera. Curr Biol. 27: 1013-1018.

1178 Pomerantz AF, Hoy MA, Kawahara AY. 2015. Molecular characterization and

1179 evolutionary insights into potential sex-determination genes in the western orchard

1180 predatory mite Metaseiulus occidentalis (Chelicerata: Arachnida: Acari:

1181 Phytoseiidae). J Biomol Struct Dyn. 33: 1239-1253.

1182 R Core Team. 2020. R: A language and environment for statistical computing. R

1183 Foundation for Statistical Computing.

1184 Rideout EJ, Narsaiya MS, Grewal SS. 2015. The sex determination gene transformer

1185 regulates male-female differences in Drosophila body size. PLoS Genet. 11:

$1186 \quad$ e1005683.

1187 Robinson MD, McCarthy DJ, Smyth GK. 2010. edgeR: a Bioconductor package for

1188 differential expression analysis of digital gene expression data. Bioinformatics 26:

1189 139-140.

1190 Romero-Pozuelo J, Foronda D, Martín P, Hudry B, Merabet S, Graba Y, Sánchez-

1191 Herrero E. 2019. Cooperation of axial and sex specific information controls

1192 Drosophila female genitalia growth by regulating the Decapentaplegic pathway.

1193 Dev Biol. 454: 145-155. 
1194

1195

1196

1197

1198

1199

1200

1201

1202

1203

1204

1205

1206

1207

1208

1209

1210

1211

1212

1213

1214

Roth A, Vleurinck C, Netschitailo O, Bauer V, Otte M, Kaftanoglu O, Page RE, Beye M. 2019. A genetic switch for worker nutrition-mediated traits in honeybees. PLOS Biol. 17: e3000171.

Rousset A., Bitsch C. 1993. Comparison between endogenous and exogenous yolk proteins along an ovarian cycle in the firebrat Thermobia domestica (Insecta, Thysanura). Comp Biochem Physiol B Biochem Mol Biol. 104: 33-44.

Sato Y, Shinka T, Sakamoto K, Ewis AA, Nakahori Y. 2010. The male-determining gene $S R Y$ is a hybrid of $D G C R 8$ and $S O X 3$, and is regulated by the transcription factor CP2. Mol Cell Biochem. 337: 267-275.

Shukla JN, Palli SR. 2012. Doublesex target genes in the red flour beetle, Tribolium castaneum. Sci Rep. 2: 948.

Sinclair AH, Berta P, Palmer MS, Hawkins JR, Griffiths BL, Smith MJ, Foster JW, Frischauf AM, Lovell-Badge R, Goodfellow PN. 1990. A gene from the human sex-determining region encodes a protein with homology to a conserved DNAbinding motif. Nature 346: 240-244.

Suzuki MG, Funaguma S, Kanda T, Tamura T, Shimada T. 2003. Analysis of the biological functions of a doublesex homologue in Bombyx mori. Dev Gene Evol. 213: $345-354$.

Takaku Y, Suzuki H, Ohta I, Ishii D, Muranaka Y, Shimomura M, Hariyama T. 2013. A thin polymer membrane, nano-suit, enhancing survival across the continuum between air and high vacuum. Proc Natl Acad Sci USA. 110:7631-7635. 
1215

1216

1217

1218

1219

1220

1221

1222

1223

1224

1225

1226

1227

1228

1229

1230

1231

1232

1233

1234

1235

1236

Takahashi M, Okude G, Futahashi R, Takahashi Y, Kawata M. 2021. The effect of the doublesex gene in body colour masculinization of the damselfly Ischnura senegalensis. Biol Lett. 17: 20200761.

Takahashi M, Takahashi Y, Kawata M. 2019. Candidate genes associated with color morphs of female-limited polymorphisms of the damselfly Ischnura senegalensis. Heredity 122: 81-92.

Takehana Y, Matsuda M, Myosho T, Suster ML, Kawakami K, Shin-I T, Kohara Y, Kuroki Y, Toyoda A, Fujiyama A, et al. 2014. Co-option of Sox3 as the maledetermining factor on the Y chromosome in the fish Oryzias dancena. Nat Commun. 5: 4157.

Taylor JS, Raes J. 2004. Duplication and divergence: the evolution of new genes and old ideas. Annu. Rev. Genet. 38: 615-643.

Thongsaiklaing T, Passara H, Nipitwathanaphon M, Ngernsiri L. 2018. Identification and characterization of doublesex from the pumpkin fruit fly, Bactrocera tau (Diptera: Tephritidae). European Journal of Entomology 115: 602-613.

True JR, Haag ES. 2001. Developmental system drift and flexibility in evolutionary trajectories. Evol. Dev. 3: 109-119.

Tunyasuvunakool K, Adler J, Wu Z, Green T, Zielinski M, Žídek A, Bridgland A, Cowie A, Meyer C, Laydon A, et al. 2021. Highly accurate protein structure prediction for the human proteome. Nature 596: 590-596.

Untergasser A, Cutcutache I, Koressaar T, Ye J, Faircloth BC, Remm M, Rozen SG. 2012. Primer3--new capabilities and interfaces. Nucleic Acids Res. 40: e115. 
1237

1238

1239

1240

1241

1242

1243

1244

1245

1246

1247

1248

1249

1250

1251

1252

1253

1254

1255

1256

Velasque M, Qiu L. Mikheyev AS. 2018. The Doublesex sex determination pathway regulates reproductive division of labor in honey bees. bioRxiv doi: 10.1101/314492.

Verhulst EC, van de Zande L. 2015. Double nexus-Doublesex is the connecting element in sex determination. Brief Funct Genom. 14: 396-406.

Wang Y, Chen X, Liu Z, Xu J, Li X, Bi H, Andongma AA, Niu C, Huang Y. 2019. Mutation of doublesex induces sex-specific sterility of the diamondback moth Plutella xylostella. Insect Biochem. Mol. Biol. 112: 1138.

Wang Y, Rensink A, Fricke U, Riddle MC, Trent C, van de Zande L, Verhulst EC. 2020. Sexually dimorphic traits and male-specific differentiation are actively regulated by Doublesex during specific developmental windows in Nasonia vitripennis. bioRxiv doi: 10.1101/2020.04.19.048553.

Wexler J, Delaney EK, Belles X, Schal C, Wada-Katsumata A, Amicucci MJ, Kopp A. 2019. Hemimetabolous insects elucidate the origin of sexual development via alternative splicing. eLife 8: e47490.

Wexler JR, Plachetzki DC, Kopp A. 2014. Pan-metazoan phylogeny of the DMRT gene family: a framework for functional studies. Dev Gene Evol. 224: 175-181.

Wiegmann BM, Trautwein MD, Winkler IS, Barr NB, Kim JW, Lambkin C, Bertone MA, Cassel BL, Bayless KM, Heimberg AM, et al. 2011. Episodic radiations in the fly tree of life. Proc Natl Acad Sci USA. 108: 5690-5695. 
1257 Wilkins AS. 1995. Moving up the hierarchy: a hypothesis on the evolution of a

1258 genetic sex determination pathway. Bioessays 17: 71-77.

1259 Xu J, Zhan S, Chen S, Zeng B, Li Z, James AA, Tan A, Huang Y. Sexually dimorphic

1260 traits in the silkworm, Bombyx mori, are regulated by doublesex. 2017. Insect

$1261 \quad$ Biochem Mol Biol. 80: 42-51.

1262 Yang Y, Zhang W, Bayrer JR, Weiss MA. 2008. Doublesex and the regulation of

1263 sexual dimorphism in Drosophila melanogaster: structure, function, and

1264 mutagenesis of a female-specific domain. J Biol Chem. 283: 7280-7292.

1265 Zhang SQ, Che LH, Li Y, Liang D, Pang H, Ślipiński A, Zhang P. 2018. Evolutionary

1266 history of Coleoptera revealed by extensive sampling of genes and species. Nat

1267 Commun. 9: 1-11.

1268 Zhuo JC, Hu QL, Zhang HH, Zhang MQ, Jo SB, Zhang CX. 2018. Identification and

1269 functional analysis of the doublesex gene in the sexual development of a

1270 hemimetabolous insect, the brown planthopper. Insect Biochem Mol Biol. 102: 31-

$1271 \quad 42$. 